\title{
Das Mausoleum von Belevi
}

Das Mausoleum von Belevi ist in seiner Substanz einer der besterhaltenen, monumentalen hellenistischen Grabbauten (Abb. 1).

\section{Zur Genese des ProjeKTes*}

Seit dem Jahre 1906, als Josef KeIL - zu diesem Zeitpunkt Sekretär der AuBenstelle des Österreichischen Archäologischen Institutes (ÖAI) in Smyrna/ İzmir und dort Nachfolger von RUdolf HEBERDEY - das ca. 14 km nördöstlich

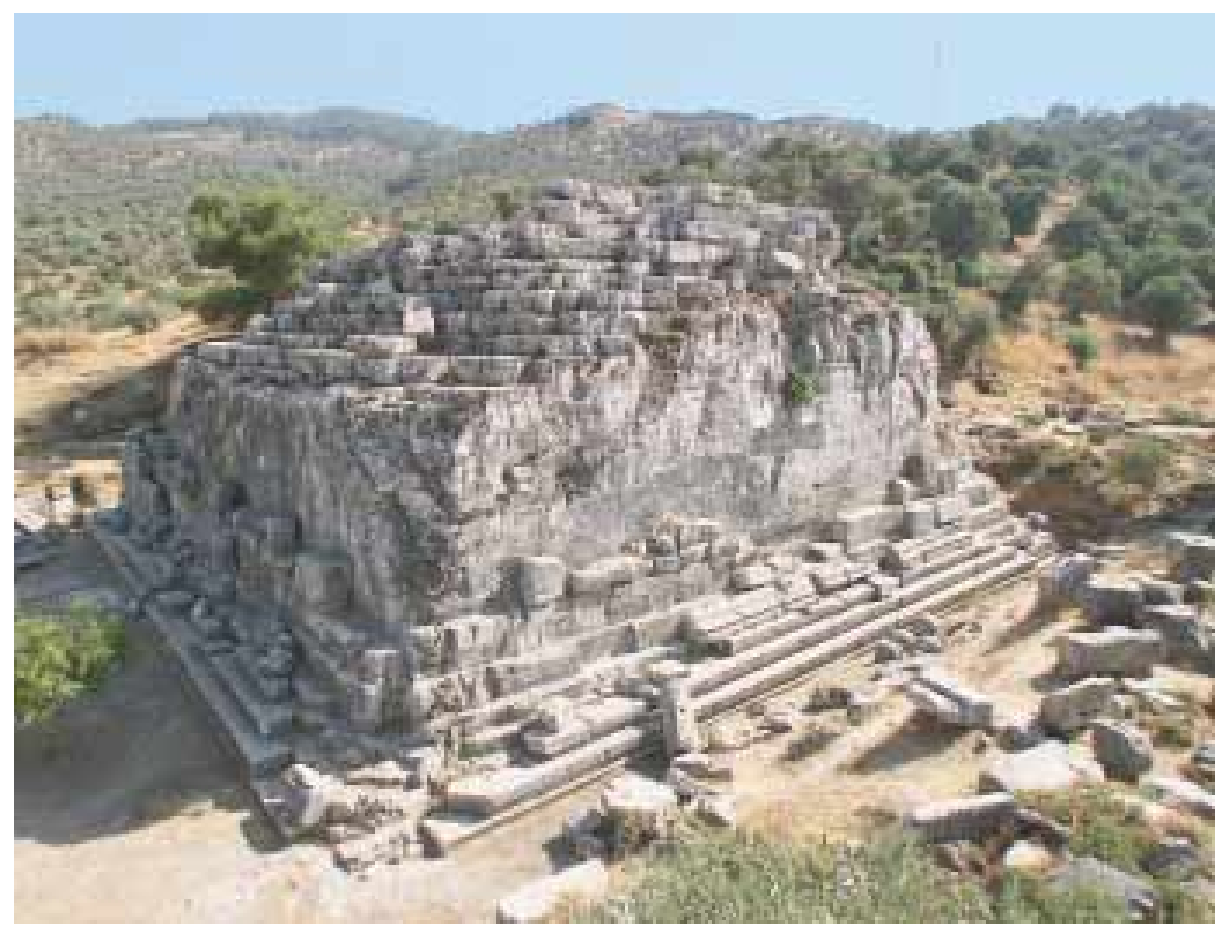

Abb. 1: Ansicht des Mausoleums von Nordosten

* Die ÖAW ist Träger des Projektes und hat die Finanzierung der archäologischen und kunsthistorischen Arbeiten übernommen. Die Bauforschung wird durch den Fonds zur Förderung der wissenschaftlichen Forschung (FWF) abgedeckt. Die Projektleitung liegt in den Händen von F. Krinzinger. Projektmitarbeiter sind P. Ruggendorfer (Archäologe; stellvertr. Leitung vor Ort) und R. Heinz (Bauforscher).

Außer den im AA 1997, 611 ff. vorgeschlagenen Sigeln des DAI werden hier folgende Abkürzungen verwendet:

Hoepfner $\quad$ W. Hoepfner, Zum Mausoleum von Belevi, AA 1993, $111 \mathrm{f}$.

Martini W. Martini, Rez. zu C. Praschniker - M. Theuer, Das Mausoleum von Belevi, FiE VI (1979), Gymnasium 88, 1981, $72 \mathrm{ff}$.

Pinkwart D. Pinkwart, Rez. zu C. Praschniker - M. Theuer, Das Mausoleum von Belevi, FiE VI (1979), BJb 183, 1983, $764 \mathrm{ff}$. 
von Selçuk liegende Monument besuchte, steht das Mausoleum von Belevi im direkten Blickpunkt der österreichischen Forschungen in Ephesos ${ }^{1}$. Im Lebenswerk von KEIL nimmt das Mausoleum von Belevi neben seinen Forschungen in Ephesos eine vorrangige Stellung ein. Dies spiegelt sich in der Einleitung seines Vortrages wider, den er am 9. Februar 1949 in der Sitzung der philosophisch-historischen Klasse der Österreichischen Akademie der Wissenschaften (ÖAW) gehalten hat: „... seit dem Jahre 1905, da ich seine Trümmer auf meinem ersten Ritt nach Lydien besuchte, hat es meine Phantasie immer wieder beschäftigt, bis mir schließlich die türkische Regierung durch Erstreckung unserer Grabungserlaubnis für Ephesos auch auf Belevi die Erfüllung meines Jugendtraumes ermöglichte"2.

Gleichzeitig findet KEIL - 14 Jahre nach Abschluß der ersten Forschungen vor Ort - klare Worte für die Verzögerungen, die mit der Publikation der Ergebnisse verbunden sind. Da für den Druck aufgrund der prekären finanziellen Situation des ÖAI die Mittel fehlen, „mußte der Weg vorläufiger, später durch Vorlage des gesamten Materials zu ergänzender Mitteilungen im Anzeiger der Akademie gewählt werden, damit die internationale Wissenschaft so rasch als möglich über den Stand der Erforschung eines Denkmals unterrichtet werde, das zweifellos in der Geschichte der griechischen Baukunst einen ersten Platz einzunehmen berufen ist"3.

Die Manuskripte von CAmillo Praschniker, der die archäologische Dokumentation und die Bearbeitung der Bauplastik übernommen hatte, und von Max Theuer, der für die Bauforschung und die Entwicklung von Rekonstruktionsvorschlägen verantwortlich zeichnete, waren trotz der schwierigen politischen Situation während und nach dem zweiten Weltkrieg weit fortgeschritten. Wesentliche Abschnitte waren jedoch noch nicht ausgearbeitet.

Als im Jahre 1949 sowohl PrAschNiker als THEUER unerwartet und in rascher Folge verstarben, traten neben diesen Problemen die Schwierigkeiten bei der weiteren redaktionellen Betreuung der vorliegenden Manuskripte speziell die Zusammenführung und Abstimmung der einzelnen Manuskriptteile und die Fertigstellung des Planmaterials - hinzu. Es war die unermüdliche Energie von KEIL und das Verdienst der nachfolgenden Direktoren des ÖAI, OtTo WALter und Fritz Eichler, daß die redaktionellen Arbeiten am Manuskript durch Egon Braun, Otto Schottenhaml, Elisabeth FosselPeschl weitergeführt werden konnten. Hermann Vetters, der selbst im Jahre 1960 eine Nachuntersuchung von geringem Umfang am Mausoleum durchführte, schließlich ist es zu verdanken, daß die Ergebnisse nach weiteren punktuellen Forschungen vor Ort und der Überarbeitung des Materials durch Wilhelm Alzinger, Robert Fleischer, Egon Reuer und Veronika

Praschniker - Theuer C. Praschniker - M. Theuer, Das Mausoleum von Belevi mit ergänzenden Beiträgen von W. Alzinger, R. Fleischer, E. Fossel-Peschl, V. Mitsopoulos, E. Reuer und O. Schottenhaml, FiE VI (1979).

Rumscheid

F. Rumscheid, Untersuchungen zur kleinasiatischen Bauornamentik des Hellenismus (1994).

1 J. Keil, ÖJh 28, 1933, 28.

2 J. Keil, AnzWien 86, 1949, 52. Zur Datierung des Besuchs in das Jahr 1905 vgl. ders., ÖJh 28, 1933, 28: „Ich hatte die beiden Denkmäler (den Tumulus und das Mausoleum, Anm. Verf.) ... bereits im zweiten Jahr meiner Stationierung in Smyrna (1906) aufgesucht und seither den Wunsch in mir getragen, das Tumulusgrab ganz auszuräumen und an der Stelle des zweifellos der hellenistischen Periode angehörigen „Heiligtums“ (= Mausoleum, Anm. Verf.) eine den Befund klärende Ausgrabung vorzunehmen."

3 J. Keil, AnzWien 86, 1949, 51 Anm. 1. 


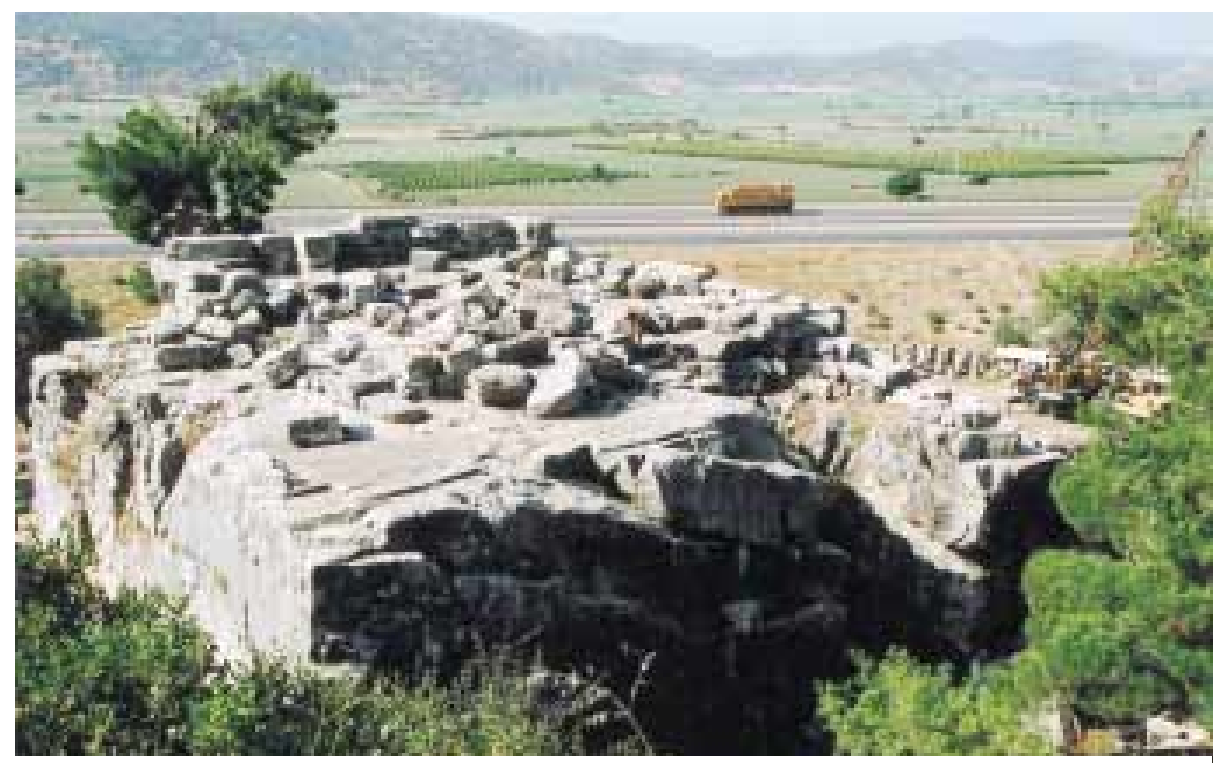

Abb. 2: Ansicht des Mausoleums von Südwesten mit der Autobahntrasse im Hintergrund

Mitsopoulos-Leon im Jahre 1979 im sechsten Band der ,Forschungen in Ephesos' vorgelegt werden konnten ${ }^{4}$.

Trotz dieser verdienstvollen und grundlegenden Publikation müssen die wissenschaftlichen Untersuchungen vor Ort und die Arbeiten an der Rekonstruktion des Monumentes als nicht abgeschlossen betrachtet werden ${ }^{5}$. In den letzten Jahren hat darüber hinaus die Gefährdung des Bestandes des Mausoleums durch den Bau der Autobahn İzmir - Aydın, deren Trasse sehr nahe (etwa $80 \mathrm{~m}$ Abstand bis zum Fahrbahnrand, ca. $60 \mathrm{~m}$ bis zum Böschungsfuß) an das Monument herangelegt wurde, erheblich zugenommen (Abb. 2). Daher entschloß man sich im Jahr 2000, die Untersuchungen fortzuführen und das Mausoleum von Belevi in das Forschungsprogramm des neu gegründeten Instituts für Kulturgeschichte der Antike an der ÖAW aufzunehmen ${ }^{6}$.

Das Projekt ist zunächst auf drei Jahre ausgelegt und soll nach weiteren zwei Jahren zu einer abgeschlossenen Publikation führen.

\section{Forschungsgeschichte}

Das Mausoleum und der Tumulus von Belevi wurden bereits im Jahre 1827 von A. Ritter von Prokesch-Osten in der Beschreibung seines Rittes von Aydın nach Smyrna erwähnt ${ }^{7}$. Knapp und präzise beschreibt der junge Offizier ${ }^{8}$ das stark verschüttete Mausoleum und schloß einen ersten Datierungsvorschlag an: „Einen niedrigen Felsblock am Fuße des Berges, wo die Hügel sich in die Ebene

4 Vgl. zu den Arbeiten am Endmanuskript das Vorwort von H. Vetters in Praschniker Theuer $5 \mathrm{ff}$.

5 Vgl. Martini 73 f.; Pinkwart 772; Hoepfner 111.

${ }^{6}$ Für die vertrauensvolle Förderung des Projektes und die Zurverfügungstellung erheblicher Mittel sei dem Präsidium und der phil.-hist. Klasse der ÖAW herzlich gedankt.

7 A. Ritter von Prokesch-Osten, Denkwürdigkeiten und Erinnerungen aus dem Orient III (1837) $461 \mathrm{f}$.

8 A. Ritter von Prokesch-Osten gilt als ein „hochgebildeter Mann von weiten geistigen Interessen“ und „Pionier der Erforschung Kleinasiens“, vgl. A. von Premerstein, Österreichische Monatsschrift für den Orient 40, 1914, 205. 
vorstrecken, sieht man nach allen Seiten behauen und unzugänglich gemacht, darauf aber einen Bau aus schweren Werkstücken, ohne Mörtel gesezt, den ich für ein kleines Schloß zur Vertheidigung der Gegend oder für das Schatzgewölbe irgend eines Herrschers oder Statthalters aus den Jahrhunderten von der Eroberung des Landes durch die Perser bis auf diejenigen durch die Römer halte. "9

Ebenfalls in der ersten Hälfte des 19. Jhs. fertigte P. TRÉMaux erste Photolithographien der Ruinen von Belevi $a^{10}$. Die ingesamt drei Aufnahmen zeigen den Tumulus, das Mausoleum von Südwesten ${ }^{11}$ sowie eine Ansicht des Mausoleums und der Terrassenmauer von Osten.

Im Jahr 1879 besuchte A. H. SAYCE auf seiner Reise entlang der kleinasiatischen Küste Belevi; im Mausoleum erkannte er die Überreste „of a Greek temple which has not been noticed before" und vermutete, daß dieser zur weiter östlich im Kaystrostal gelegenen Stadt Larissa gehört habe ${ }^{12}$.

1880 veröffentlichte G. WEBER in der Zeitschrift der evangelischen Schule in İzmir erste detaillierte Beschreibungen beider Monumente und einiger verstreut um das Mausoleum liegender Architekturfragmente. Auch er deutete das Mausoleum als Heiligtum ${ }^{13}$.

Den Angaben von Weber folgend bezeichneten G. Perrot und Ch. CHIPIEz 1890 das Mausoleum gleichfalls als Heiligtum. Als Datierung führten sie „l'epoque romaine“ an und bildeten den Tumulus anhand der von Weber publizierten Pläne $a b^{14}$.

Bei einer Begehung des „Heiligtums von Belevi südöstlich der Bahnstation Kos-Bunar" entdeckte E. S. JoRDANIDIS 1898 vor der Ostseite des Mausoleums den Architravblock mit der $\mathrm{H} \Lambda \mathrm{IA} \Delta \mathrm{E} \Sigma$-Inschrift ${ }^{15}$.

Der Bericht von WeBer veranlaßte schließlich KeIL, im Jahre 1906 die Ruinen von Belevi zu besuchen ${ }^{16}$.

Erst im Jahre 1931 konnte KeIL, unterstützt durch F. MiLtNeR, im Rahmen der "Grabungslizenz Ephesos" eine viertägige Voruntersuchung des Mausoleums und des benachbarten Tumulus durchführen ${ }^{17}$. Trotz der kurzen Zeit gelang es, die Bestimmung und Architektur des Monumentes in den Grundzügen zu erkennen. Eine erste Rekonstruktion, welche die wesentlichen Elemente des Monumentes - bestehend aus Sockel- und Obergeschoß wiedergibt, wurde in einem Vorbericht von MiLtner vorgelegt. Gleichzeitig wurden erste Überlegungen zur Datierung und zum Grabherrn publiziert ${ }^{18}$.

In den Jahren 1933 und 1935 befaßten sich die Ephesos-Kampagnen u. a. finanziert durch Spenden der Rockefeller-Foundation - intensiv mit dem Mausoleum ${ }^{19}$. Der Sockel, der v. a. an der Südseite hoch verschüttet war, sowie die Grabkammer und der nähere Bereich um das Monument wurden freigelegt.

9 von Prokesch-Osten a. O. $461 \mathrm{f}$.

10 P. Trémaux, Exploration archéologique en Asie Mineure (o. J.).

11 Vgl. Praschniker - Theuer Abb. 139.

12 A. H. Sayce, JHS 1, 1880, 75 ff. bes. 91 .

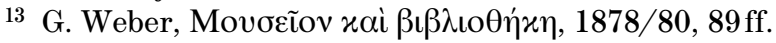

14 G. Perrot - C. Chipiez, Histoire de l'art dans l'antiquité V. Perse - Phrygie - Lydia et Caria - Lycia (1890) $280 \mathrm{ff}$.

15 E. S. Jordanidis, AM 23, 1898, 165.

16 J. Keil, AnzWien 86, 1949, $51 \mathrm{f}$.

17 Auch die nun am Monument unternommenen Forschungen und Grabungen werden im Rahmen der „Grabungslizenz Ephesos“ durchgeführt.

18 J. Keil, ÖJh 28, 1933, Beibl. $27 \mathrm{ff}$.

19 J. Keil, ÖJh 29, 1935, Beibl. 105ff.; ders., ÖJh 30, 1937, Beibl. 175 ff. Im Jahre 1934 wurden weder in Ephesos noch in Belevi Grabungen durchgeführt, T. Wohlers-Scharf, Die Forschungsgeschichte von Ephesos. Entdeckungen, Grabungen und Persönlichkeiten (1995) 119. 
Die Nachuntersuchung, die H. VetTers im Jahre 1960 am Mausoleum durchführte, beschränkte sich auf die Aufnahme der im Süden des Monumentes vorbeiführenden Wasserleitung ${ }^{20}$.

1965 begann S. KASPER mit den Grabungen am benachbarten älteren Tumulus, als dessen Grabherr der Heros Pixodaros, der Entdecker der Steinbrüche von Belevi, vermutet wird ${ }^{21}$.

In den Jahren 1974-78 erfolgte in einem weiteren großen Anlauf schließlich die gründliche Überarbeitung der Architektur und Plastik des Mausoleums durch W. Alzinger und R. Fleischer, wobei auch Grabungen auf der Terrasse im Osten des Mausoleums durchgeführt wurden. Die Keramik und die Knochenfunde aus den Altgrabungen der 30er Jahre wurden von V. MiтSOPOULOS-LEON und von E. REUER neu bearbeitet ${ }^{22}$.

Gleichfalls in den 70er Jahren wurde zum Mausoleum am Institut für Klassische Archäologie der Universität Ankara geforscht. Betreut von E. Akurgal verfaßte S. Buluç (†) ihre Dissertation über das Mausoleum ${ }^{23}$.

1993 hat W. HoEPFNER einen Rekonstruktionsvorschlag, der auf einem Ansatz von Theuer beruht, für das Obergeschoß des Monuments vorgelegt ${ }^{24}$.

In den Jahren 1998 und 1999 wurden vom ÖAI in Kooperation mit dem Institut für Klassische Archäologie der Universität Wien Dokumentationskampagnen vor Ort durchgeführt, die der Projektvorbereitung und der Aktualisierung der Fragestellungen dienten ${ }^{25}$.

Während der Kampagne 2000 wurde mit Grabungen vor der Ostseite des Mausoleums begonnen ${ }^{26}$.

20 F. Eichler, AnzWien 98, 1961, $65 \mathrm{ff}$.

21 S. Kasper, ÖJh 51, 1976-77, Beibl. $127 \mathrm{ff}$.

22 Vgl. Praschniker - Theuer $161 \mathrm{ff} .201 \mathrm{f}$.

${ }^{23}$ Die Arbeit wurde nicht veröffentlicht, vgl. allerdings S. Buluç in: The Proceedings of the Xth International Congress of Classical Archaeology (1978) $1085 \mathrm{ff}$.

${ }^{24}$ Hoepfner $111 \mathrm{ff}$.

25 Schon in den Jahren zuvor wurde auf Anregung von J. Borchhardt der Versuch gestartet, die Neuuntersuchungen des Monumentes im Rahmen eines EUROCAREProjektes zu organisieren. Einige Begehungen wurden durchgeführt. Als Vorleistung des ÖAI wurde 1998 das Terrain entlang der staatlichen Grundstücksgrenzen um das Mausoleum als erste Maßnahme zur Abgrenzung einer archäologischen Zone und zum Schutz des Monumentes vor zunehmenden Vandalismus, Steinraub und anderen schädigenden äußeren Einflüssen eingezäunt. Die dafür erforderlichen vermessungstechnischen Arbeiten wurden vom Museum von Selçuk durchgeführt. Außerdem begann man mit der Erstellung eines topographischen Planes, der den Verlauf der Autobahntrasse im Bereich des Mausoleums sowie die Lage des benachbarten Tumulus dokumentiert. Neben der photographischen Dokumentation des topographisch stark veränderten Terrains rund um das Mausoleum erfolgte eine Sichtung und Überprüfung des Zustandes des Monumentes und der ausgelegten Bauglieder. Auf dem Sockel des Mausoleums und im Nahbereich des Monumentes wurde mit der Installierung eines Vermessungsnetzes begonnen. Zur Dokumentationskampagne 1998 und 1999 vgl. die Vorberichte von R. Heinz - G. Kaymak - P. Ruggendorfer, ÖJh 68, 1999, Beibl. Jahresbericht 1998, $31 \mathrm{ff}$. und ÖJh 69, 2000, Beibl. Jahresbericht 1999, $377 \mathrm{f}$.

${ }^{26}$ Mitarbeiter der Kampagne 2000 waren neben den Verfassern Frau DI Dr. Gamze Kaymak (Architektin), Herr DI Stefan Klotz (Geodät), Frau Mag. Hannah Liko (Archäologin), Herr Christian Schirmer (Geodät) und Frau Andrea Sulzgruber (Photographin). 


\section{Die Platzanlage}

Nördlich des Monumentes verlief der antike Weg, der Ephesos und Sardes über das Tmolon-Gebirge verbunden hat. Dieser Weg entspricht möglicherweise dem Verlauf jener Prozessionsstraße, die vom Artemision von Ephesos zu dem von diesem aus gegründeten Heiligtum der Artemis in Sardes führte ${ }^{27}$. Die Trassenführung des Weges ist unbekannt, seine Existenz wird jedoch durch Meilensteine unterschiedlicher Zeitstellung dokumentiert ${ }^{28}$.

Östlich des Mausoleums erstreckt sich eine Platzanlage, die in ca. $48 \mathrm{~m}$ Entfernung vom Grabbau durch eine $6 \mathrm{~m}$ hohe und etwa 3,1 m starke Terrassenmauer begrenzt wird. Die Mauer ist noch auf einer Länge von $75 \mathrm{~m}$ erhalten. Mitten auf der Terrasse liegt ein Kalkofen. Südlich des Ofens konnte im Jahr 2000 eine 4,50 m lange und $4 \mathrm{~m}$ breite Sondage geöffnet werden, die zur Untersuchung der Frage nach der Gestalt und nach möglicher Bebauung der Terrasse diente.

Wie der Grabungsbefund zeigte, wurde die Terrasse mit Hilfe antiker Steinmetz- und Steinbruchtechnik aus dem natürlichen, anstehenden Felsen gewonnen und blieb wie das Monument selbst unvollendet. In etwa 2,50 m Tiefe konnten zwei Schrotkanäle freigelegt werden, wovon der nördliche etwa $0,65 \mathrm{~m}$ tief in den Felsen eingearbeitet ist. Der südlichere ist flacher gearbeitet und wurde noch in antiker Zeit durch Regenwasser stark ausgewaschen. Die Arbeiten zur Gewinnung der Terrasse wurden offensichtlich zu einem Zeitpunkt eingestellt, als der Felsen teilweise noch sehr hoch anstand, wie der Befund vor dem Ostprofil der Sondage zeigt. Hier befindet sich in etwa $15 \mathrm{~m}$ Abstand zum Sockel des Mausoleums eine 1,7 m hohe Geländekante im Felsen. Dies bedeutet, daß das Gelände vom Mausoleum Richtung Osten abgearbeitet wurde. Der Kalkofen wurde später offenbar an günstiger Stelle in den stufenartigen Geländeverlauf eingesetzt. Seine Wandung wurde in Bruchsteinmauertechnik aufgemauert und die Außenmauern mit Erdmaterial hinterfüllt.

Entlang der Südseite und teilweise auch an der Westseite des Sockels des Mausoleums hat man in der Kaiserzeit eine Wasserleitung angelegt, in der sich bereits Teile der Bauplastik - speziell von den Dachskulpturen - als Spolien verbaut fanden ${ }^{29}$.

\section{Das Monument}

Architektonisch gliedert sich der Grabbau in ein quadratisches, in dorischer Ordnung gestaltetes Untergeschoß und in ein in korinthischer Architektur ausgeführtes Obergeschoß.

\subsection{Das Untergescho $\beta$}

Der Kern des Untergeschosses wurde direkt aus dem anstehenden Kalkstein eines Geländevorsprungs herausgeschnitten. Der grob geglättete Felskern des Untergeschosses wurde mit Marmorblöcken verkleidet. Die Krepis des Sok-

27 Kasper a. O. 163.

${ }^{28}$ R. Meriç - R. Merkelbach - J. Nollé - S. Şahin (Hrsg.), Die Inschriften von Ephesos VII, 2 (1981) $305 \mathrm{ff}$.

29 Eichler a. O. 65 ff.; Praschniker - Theuer 11 Anm. 2. 


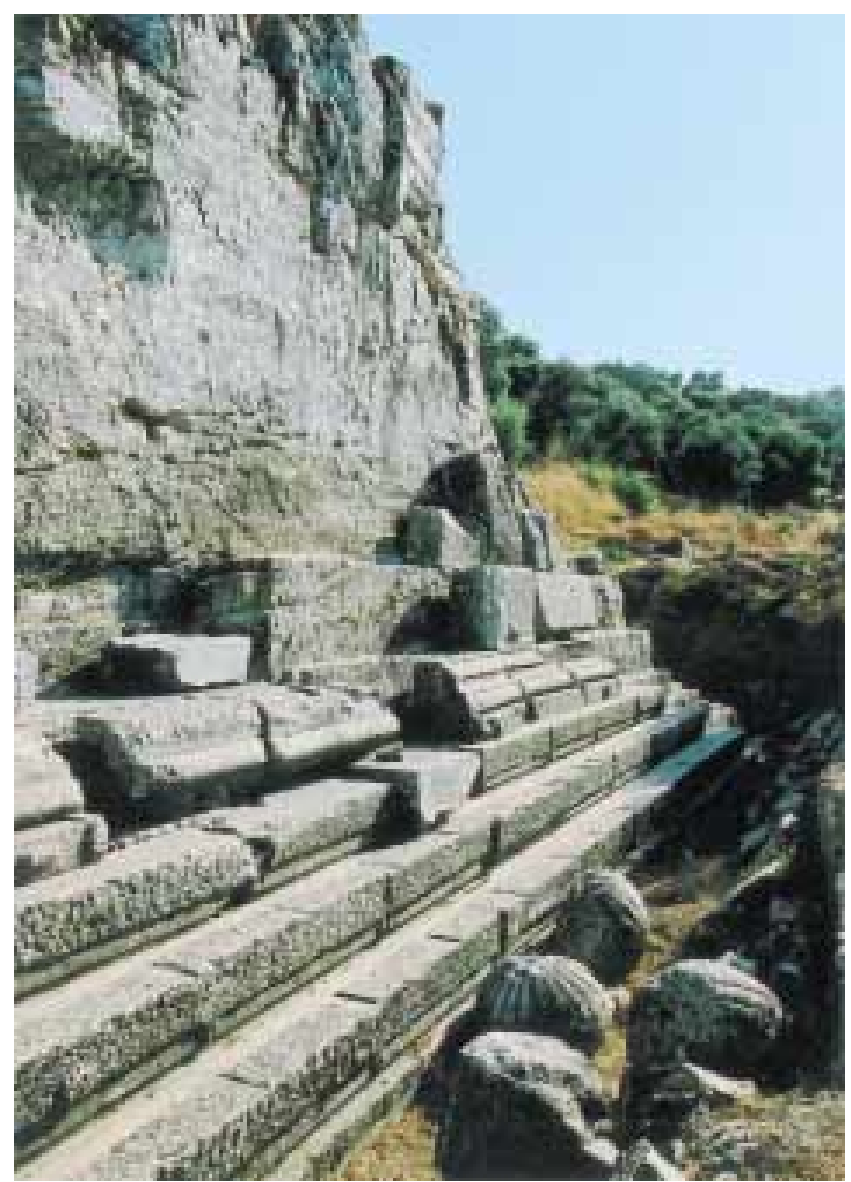

Abb. 3: Stufenunterbau, Sockelprofil und Schwelle der Scheintür an der Nordseite

kels ruht auf einer quadratischen Grundfläche von etwa 29,70 m Seitenlänge. Über dem dreistufigen Unterbau, der auf einer Euthynterie auflagert, folgt ein Fußprofil bestehend aus Plinthe, Torus, Echinus und einem zurückgesetzten, liegenden lesbischen Kymation. Durch die Wandblöcke, mit denen der grob geglättete Felskern des Sockels verkleidet wurde, hat man an den Außenseiten den Eindruck eines massiv durchgeschichteten Untergeschosses erzielt. Die obere Begrenzung war in Form eines kanonischen dorischen Gebälkes mit Architrav, Metopen-Triglyphenfries und Kranzgesims gebildet. Palmetten zierten die Eckfelder an der Unterseite des Geisons. In der Mitte der Nordseite des Sockels ist eine Scheintür eingearbeitet (Abb. 3). Wie am Fußprofil sind auch zahlreiche Details des Gebälkes unfertig geblieben und in unterschiedlichen Stadien der Ausarbeitung erhalten. Dieser Umstand gibt wesentliche Details der antiken Werk- und Fertigungstechnik wieder.

Die Grabkammer, die im Süden in den Felskern eingearbeitet ist, liegt nicht in der Mittelachse des Monuments, sondern ihre Lage ist etwas nach Osten verschoben. Sie besteht aus einer größeren Haupt- und einer kleineren Vorkammer, deren Bodenniveau um etwa $44 \mathrm{~cm}$ tieferliegt. Beide Kammern waren durch eine $Z$ Wwischenwand getrennt und verfügten über einen Bodenbelag aus rechteckig geschnittenen Platten, die mit exaktem Fugenschluß verlegt waren. Wie die Einlassungen in den Fußbodenplatten beweisen, lief der Flügel der Verbindungstür zwischen Vor- und Hauptkammer auf einer viertelkreisförmigen Metallschiene. Obwohl die Tür in Form einer monolithischen Platte gearbeitet war, scheint die Reliefierung an der der Vorkammer zuge- 
wandten Seite eine doppelflügelige Struktur mit vier Paneelen zu imitieren ${ }^{30}$. Eingedeckt war die Grabkammer mit einem Tonnengewölbe ${ }^{31}$.

Der Sarkophag stand in der Hauptkammer an der rechten Seitenwand nahe der Tür. Er befindet sich heute im Museum von Selçuk ${ }^{32}$. Zwischen dem Sarkophag und der Hinterwand der Grabkammer ist aus dem Felsen eine marmorverkleidete Bank herausgearbeitet, deren Höhe und Tiefe jener des Sarkophagkastens entspricht.

Die Quaderverkleidung des Felssockel schloß die Grabkammer nach außen vollständig ab, so daß diese für den antiken Betrachter nicht erkennbar war. In der Innenkonstruktion der Krepis blieb eine dromosartige Öffnung ausgespart, die den Zugang in die Grabkammer bis zum Zeitpunkt der Bestattung ermöglichte und erst danach verschlossen wurde. Möglicherweise wurde diese Öffnung auch bei einer Zweitbelegung oder Beraubung des Grabes genutzt, wie dies spätere Keramikfragmente nahelegen, die in der Grabkammer außerhalb des Sarkophages gefunden wurden ${ }^{33}$.

\subsection{Das Obergescho $\beta$}

Das Obergeschoß wird durch einen rechteckigen Kernbau mit umlaufender Säulenhalle gebildet. Die kannelierten Säulen des Umgangs erhoben sich auf attischen Basen und wurden von korinthischen Kapitellen bekrönt. Die zentralen Felder der Kassettendecke der Peristasis waren mit figuralen Reliefdarstellungen geschmückt. Diese waren ebenso wie die Kapitelle und das Gebälk polychrom bemalt. Die zahlreichen marmornen Dacheindeckungsplatten, die sich um das Monument verstreut fanden, sind als Eindeckung des Peristasisdaches zu interpretieren ${ }^{34}$.

Unterschiedlich gearbeitete Fragmente eines Epistyls mit zwei Faszien und geringer dimensionierte Säulentrommeln mit nur zu zwei Drittel ausgearbeiteten Kanneluren sowie Palmblattkapitelle sind der Architektur des Obergeschosses zuzuzählen. Diese Teile haben bei den einzelnen Rekonstruktionen unterschiedliche Berücksichtigung und Zuweisung erfahren.

Einige der Zwei-Fascien-Architrave tragen Inschriftenreste, von denen neben den Buchstabengruppen EY $\Sigma$ und $\Phi$ A nur das Wort H $\Lambda$ IA $\Delta \mathrm{E} \Sigma$ auf einem Block zur Gänze erhalten ist ${ }^{35}$. Auf einem weiteren Bruchstück ist nur

30 Vgl. die Gestaltung der Türen bei makedonischen Gräbern, B. Gossel, Makedonische Kammergräber (1980) 54f.; S. G. Miller, The Tomb of Lyson and Kallikles. A Painted Makedonian Tomb (1993) 8f.

31 Praschniker - Theuer 61. W. Hoepfner, AA 1996, 95 ff. bes. 110 vermutet, daß die Grabkammer des Maussolleions von Halikarnassos gleichfalls über ein Tonnengewölbe verfügte. Hingegen spricht sich K. Jeppesen, JdI 107, 1992, 66 ff. für ein Kraggewölbe in Halikarnassos aus.

32 Zur ungewöhnlichen Wahl des Aufstellungsortes vgl. R. Fleischer, Studien zur seleukidischen Kunst 1. Herrscherbildnisse (1991) 24: „Es fällt auf, daß sich die gelagerte Darstellung von links dem Beschauer viel besser erschließt als von rechts. Von links nähern sich auch die Adoranten auf den Heroenreliefs dem gelagerten Heros, während der in die Grabkammer von Belevi Eintretende von der anderen Seite kam. Man hätte dieser Schwierigkeit begegnen können, indem man den Sarkophag an die andere Langseite der Kammer stellte, doch war der Platz schon durch die erste Bauperiode für Lysimachos vorgegeben, die nach der Ausgestaltung des Sarkophagkastens keinen Deckel mit gelagerter Figur vorsah. "

${ }^{33}$ Vgl. Abschnitt 6.

34 Praschniker - Theuer 53f.; Hoepfner 114.

$35 \mathrm{Zu}$ den Inschriftenfragmenten vgl. Praschniker - Theuer 47. 
noch ein $\Phi$ und eine gerade Haste erkennbar, die von KeIL als Ansatz des Buchstaben Rho interpretiert wurde ${ }^{36}$.

Vom Kernbau haben sich in mehreren Schichten die Reste einer aufgehenden Mauer, die parallel zur Nordseite des Sockels verlief, erhalten. Die Mauerstärke läßt sich zur Zeit nicht eindeutig bestimmen. In den bisher vorgelegten Rekonstruktionen wird in dieser Mauer in mittiger Lage eine Scheintür angenommen, die der im Untergeschoß entspricht. Unklar ist außerdem, ob der Mauer an der Innen- oder an der Außenseite eine Säulenstellung vorgelagert war ${ }^{37}$.

Klar erkennbar sind jedoch pfeilerartige Vorsprünge an der Innenseite der Mauer, deren untere Schichten im Norden noch erhalten sind. An diese Mauer ist von Süden der aus unregelmäßigen Steinen bestehende Unterbau eines Plattenbelags angesetzt ${ }^{38}$. Der Plattenbelag ist mehrfach geneigt, die Hauptrichtung des Gefälles war nach Westen orientiert. Außer den bereits erwähnten Marmordachsteinen zur Eindeckung der Peristasis haben sich keine Hinweise auf eine Dachkonstruktion erhalten ${ }^{39}$.

\subsection{Die Bauplastik}

Vom Dachschmuck haben sich Grabvasen und Löwengreifen erhalten, die jeweils antithetisch gruppiert waren. Außerdem kennen wir Torsen und Fragmente von unterlebensgroßen Pferden, die als Eckakrotere zu verstehen $\operatorname{sind}^{40}$.

Weitere anthropomorphe, rundplastische Fragmente - zwei Armfragmente, die möglicherweise von Frauenstatuen stammen, und ein Gesichtsfragment - wurden allerdings auch anderen Aufstellungsorten zugewiesen ${ }^{41}$.

Die zentralen Felder der Kassettendecke der Peristasis waren mit figuralen Reliefdarstellungen geschmückt. Während an der Nordseite diese Reliefs Szenen aus dem agonistischen Bereich wiedergeben und neben gymnischen Agonen vielleicht auch ein hippischer Wettkampf dargestellt ist, sind die Darstellungen der übrigen Seiten dem Kentaurenkampf gewidmet (Abb. 4) ${ }^{42}$. Hervorzuheben ist die Szene im Kassettenfeld in der Mitte der Nordseite (N 4), in der die zentrale bärtige und bekleidete Figur einen rechts neben ihr stehenden, nackten Wettkämpfer bekränzt, während die linke begleitende Figur ein Blasinstrument spielt. Die zentrale Figur wurde als Grabherr gedeutet $^{43}$.

Die Inschriftenreste auf den Zwei-Fascien-Architraven wurden von KeIL, Fleischer und Pinkwart mit dem Phaeton-Mythos in Zusammenhang gebracht $^{44}$. Die Buchstaben wirken als Beischriften für Malerei monumental,

36 Praschniker - Theuer 47.

37 Vgl. in Gegenüberstellung Praschniker - Theuer 57 Abb. 42 a und Hoepfner 116 Abb. 8.

38 Die Fugen waren lt. Hoepfner 114, mit einem grobem wasserundurchlässigen Kalkmörtel - vermischt mit Kieseln und Tonerde abgedichtet. Diese Aussage ist vor dem Befund heute nicht mehr nachvollziehbar.

39 Praschniker - Theuer 52; vgl. Hoepfner 111.

40 Praschniker - Theuer $89 \mathrm{ff} .142 \mathrm{ff}$.

41 R. Fleischer in: Praschniker - Theuer 148.

42 Praschniker - Theuer $73 \mathrm{ff}$. $128 \mathrm{ff}$.; K. Tancke, Figuralkassetten griechischer und römischer Steindecken (1989) $25 \mathrm{ff}$.

$43 \mathrm{Vgl}$. Fleischer a. O. 130.

44 Fleischer a. O. 148; Pinkwart 765. 


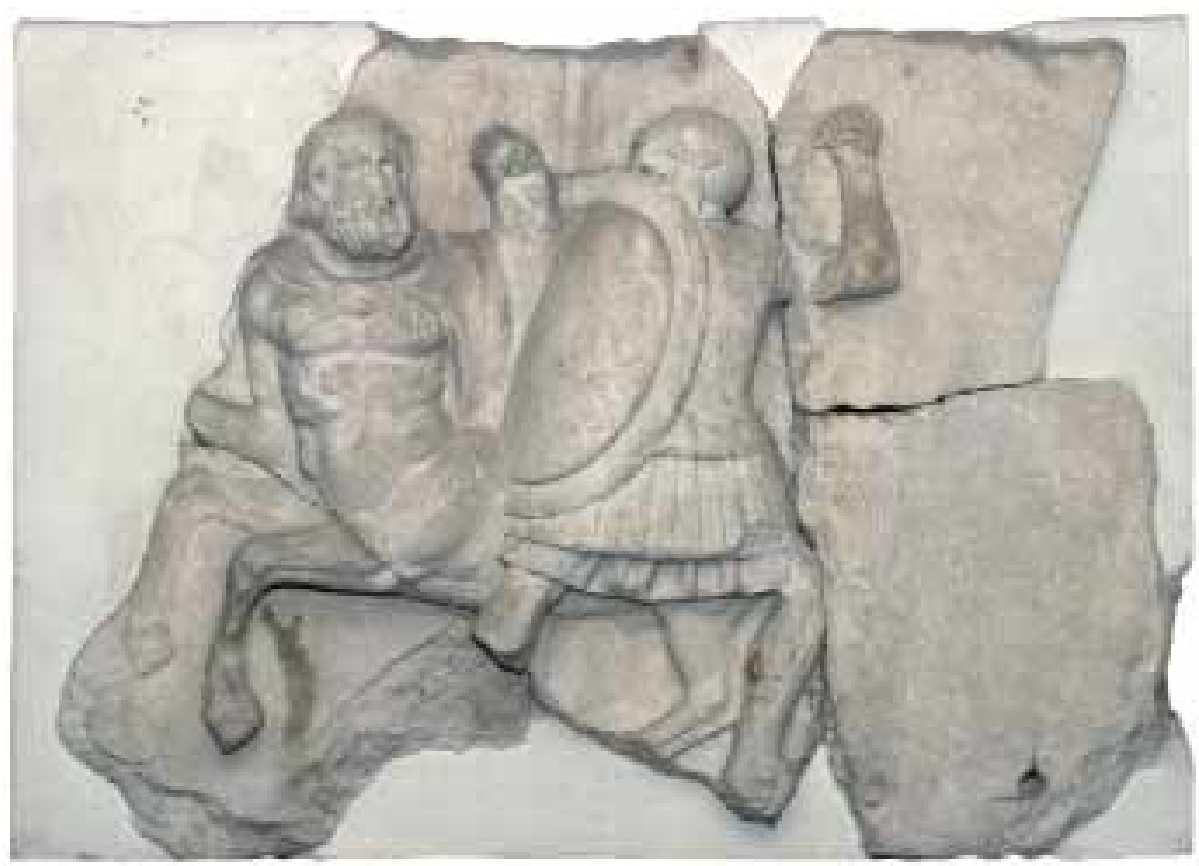

Abb. 4: Kassettenplatte mit Kentaurenkampfrelief (W 3)

ein inhaltlicher Bezug zu den Reliefs der Kassettendecke ist auszuschließen. Es bleibt die Frage, ob diese Inschriften auf eine allerdings nicht erhaltene rundplastische Darstellung des Phaeton-Mythos bezogen werden können ${ }^{45}$.

\subsection{Die Ausstattung der Grabkammer}

Der monumentale Klinensarkophag aus Marmor trägt eine nach rechts gelagerte männliche Deckelfigur, die wir als frühestes Beispiel dieses Typus im griechischen Osten in vorrömischer Zeit bezeichnen können.

Der etwa 2,58 m lange, 1,29 m breite und 0,92 $\mathrm{m}$ hohe Sarkophagkasten ist aus einem Block gefertigt. An der Vorderseite des Sarkophagkastens sind im Relief eine Kline, eine Polsterbank und ein Fußschemel wiedergegeben. Zusätzlich schmückt ein Fries in der Form von elf musizierenden Sirenen (Flöten- und Lyraspielerinnen sowie Sängerinnen) die Kline. Der Fries ist unfertig, die Figuren sind in unterschiedlichen Stadien der Ausarbeitung erhal$\operatorname{ten}^{46}$. Die beiden Seitenwände und die Rückwand des Sarkophagkastens schlossen direkt an das Mauerwerk an und sind deshalb unverziert (Abb. 5). In Inneren des Sarkophages fanden sich neben mehreren Kleintierknochen einige Knochenfragmente und zwei Zähne eines männlichen Individuums im Alter von etwa 40 bis 45 Jahren $^{47}$.

Der Sarkophagdeckel ist um $9 \mathrm{~cm}$ länger als der Kasten und aus zwei Teilen zusammengesetzt.

45 So schon J. Keil, AnzWien 86, 1949, $51 \mathrm{ff}$.

46 Generell lassen sich am Sarkophagkasten verschiedene Formen der technischen Bearbeitung feststellen, B. Schmaltz, ÖJh 49, 1968-71, 63ff.; R. Fleischer in, Praschniker - Theuer $148 \mathrm{ff}$. Abb. 118. 119.

47 E. Reuer in: Praschniker - Theuer 201. Eine Altersbestimmung der Knochenfragmente mittels moderner naturwissenschaftlicher Methoden steht zur Zeit noch aus. 


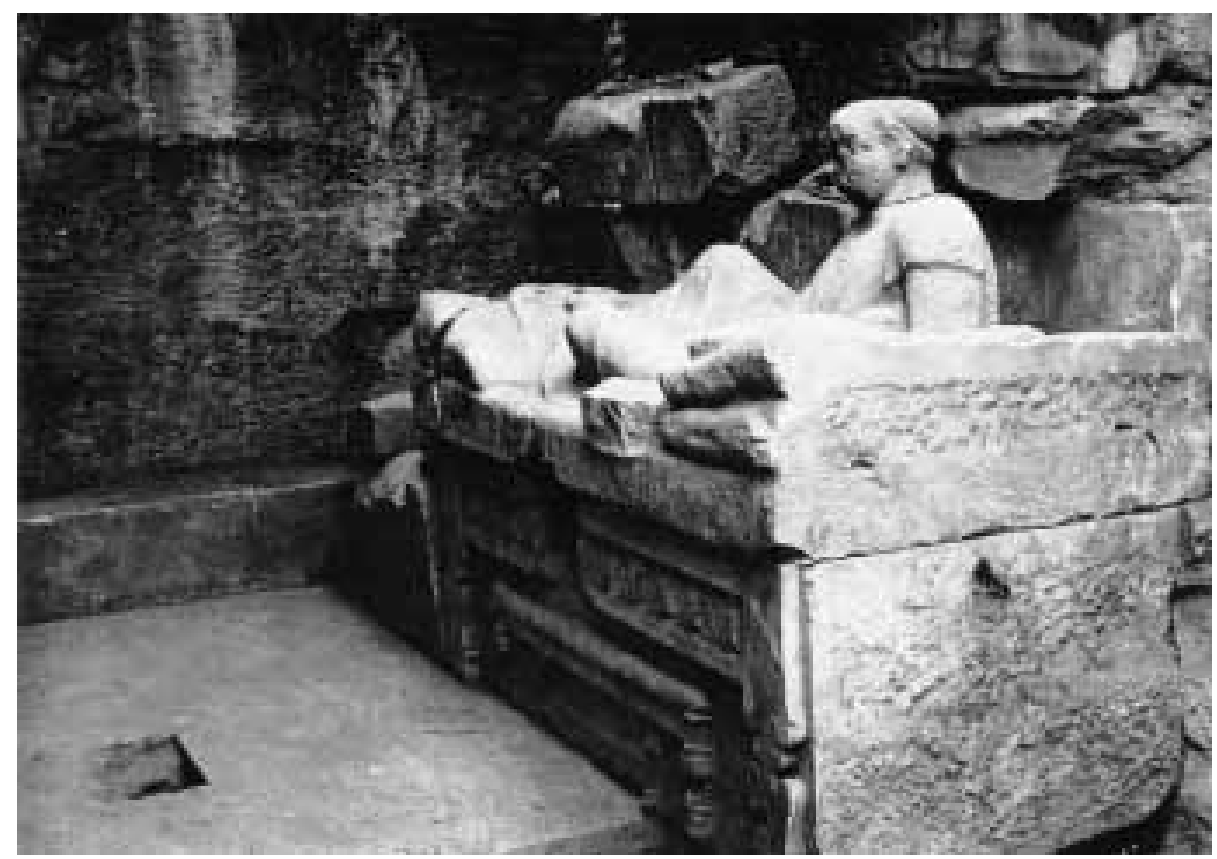

Abb. 5: Sarkophag in der Grabkammer

Die nach rechts gelagerte Figur ist auf ein Kissen gestützt, wobei der Oberkörper stark in die Vertikale gerückt ist, so daß sich im Beckenbereich ein unorganischer, der gelagerten Haltung widersprechender Knick ergibt.

Mit einer Schale in der rechten Hand und dem unterschlagenen linken Bein erinnert der Dargestellte an den in den Totenmahlreliefs ausgebildeten Typus $^{48}$. Im Gegensatz zum rechten Arm ist der Linke ab dem Ellbogenbereich nur grob in seinen Umrissen ausgearbeitet. Der Kopf des Gelagerten ist stark beschädigt und teilweise mit einer starken Kalksinterschicht überzogen. Nur das bartlose, rundliche Gesicht der Figur ist fertiggestellt und sorgfältig ausgeführt, wie die erhaltenen Details der Augen und die Flächen der Wangen zeigen. Andere Partien, wie das Haar oder v. a. das linke Ohr, sind unfertig geblieben. Die mit dem Spitzeisen grob eingeschlagene Rille am Kopf kann zur Anbringung eines Kranzes, einer Wulstbinde oder eines Diadems gedient haben ${ }^{49}$.

Der schlechte Erhaltungszustand des Kopfes und der hohe Grad seiner Verunreinigung erschweren die physiognomische Untersuchung, so daß eine Beantwortung der Frage nach der dargestellten historischen Persönlichkeit nach Kriterien der Porträtforschung zur Zeit nicht erfolgreich sein $\mathrm{kann}^{50}$. Damit ist natürlich auch die Frage nach dem Grabherrn verbunden. Von einer eindeutigen Benennung muß zur Zeit Abstand genommen werden.

48 R. Fleischer, Studien zur seleukidischen Kunst 1. Herrscherbildnisse (1991) 24.

49 Zuletzt hat sich Fleischer a. O. 24 unter Hinweis auf den V-förmigen Querschnitt der Rille, der mit einem nur leicht ins Haar drückenden Diadem nicht vereinbar sei, für eine Wulstbinde ausgesprochen. Für ein Diadem argumentieren: J. Keil, ÖJh 29, 1935, Beibl. 144; G. Kleiner, Diadochen-Gräber (1963) 84; H. Bauer, Korinthische Kapitelle des 4. und 3. Jh. v. Chr. (1973) 115; J. J. Pollitt, Art in the Hellenistic Age (1986) 290; B. Sismondo Ridgway, Hellenistic Sculpture I (1990) 195 Taf. 88.

50 Eine Reinigung des Kopfes ist für die nächsten Kampagnen geplant. 


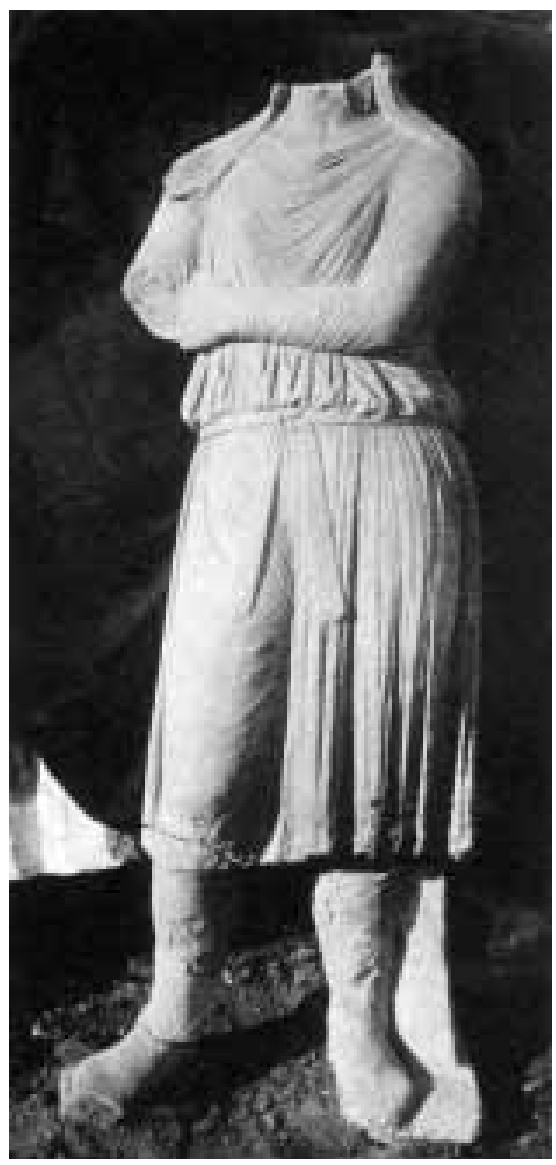

In der Grabkammer wurde während der Ausgrabungen der 30er Jahre die etwa lebensgroße Statue eines orientalisch gekleideten Mannes gefunden, die in der Mitte der Kammer vor dem Sarkophag lag und sich heute im Museum von İzmir befindet (Abb. 6) ${ }^{51}$. Beide Füße sind gebrochen, der Körper selbst ist gut erhalten $^{52}$. Ohne dem fehlenden Kopf mißt die Staue $1,56 \mathrm{~m}$. Trotz der geäußerten Vermutung, daß diese Figur zum Dachschmuck zu zählen sei ${ }^{53}$, scheint an der Zugehörigkeit der Statue zum Ausstattungsprogramm der Grabkammer kein Zweifel zu bestehen ${ }^{54}$. Der Trauergestus der Statue legt eine Interpretation als Diener- bzw. Wächterfigur nahe. Die Unterseite der Statuenplinthe ist bislang nicht untersucht worden, so daß zur Zeit weder der Aufstellungsort in der Grabkammer in Beziehung zum Sarkophag noch die technische Verbindung mit dem Untergrund geklärt werden kann ${ }^{55}$.

Abb. 6: Statue eines orientalisch gekleideten Mannes („Dienerfigur“)

${ }^{51}$ Vgl. R. Fleischer in, Praschniker - Theuer 147. Zum Gewand vgl. A. Linfert, Kunstzentren hellenistischer Zeit (1976) 23.

52 Durchbrochen waren der linke Unterschenkel samt Stütze, der rechte Fußrist und zweimal der rechte Unterschenkel. Außerdem fanden sich an der gesamten Figur Brandspuren sowie starke Versinterungen, Fleischer a. O. 146.

${ }_{53}$ Praschniker - Theuer 94.

54 Die Statue müßte, wenn sie vom Dach in die Grabkammer gestürzt wäre, stärker fragmentiert sein. Vgl. dazu die Fragmente der Dachskulpturen und der Teile der Architektur des Obergeschosses, die gleichfalls bei der Freilegung der Grabkammer gefunden wurden, aber im Gegensatz zu der orientalisch gekleideten Figur kleinteilig zerbrochen sind, Praschniker - Theuer 94. Die von B. Andreae, Schönheit und Realismus. Auftraggeber, Schöpfer, Betrachter hellenistischer Plastik (1998) 75f. vorgenommene Zuordnung einer im Museum von İzmir ausgestellten Figur eines verschränkt sitzenden Orientalen als zweite Dienerstatue aus der Grabkammer des Mausoleums von Belevi beruht offensichtlich auf einer Verwechslung. Die Statue wurde in Halikarnassos gefunden und ist mehrfach besprochen worden, vgl. U. v. WilamowitzMoellendorff - G. Karo, AM 45, 1920, $160 \mathrm{ff}$. Taf. 4, 1 sowie von H. Möbius, AM 50, 1925, 45 ff. Abb. 1; Praschniker - Theuer 95 Anm. 59. Die von Andreae a. O. 300 im Abbildungsverzeichnis für die Figur angeführte Inv.-Nr. 1085 trägt der Löwengreif Nr. 2, der bei Praschniker - Theuer 90. 142 Abb. 71.75 behandelt und wiedergegeben ist, nochmals von R. Fleischer in: Praschniker - Theuer 142 Abb. 112 besprochen wurde und heute in den Ausstellungsräumen in antithetischer Aufstellung mit einem weiteren Löwengreif um eine Marmorvase präsentiert wird. Der sitzende Orientale wird im Museum unter der Inv.-Nr. 506 geführt. Vgl. R. Heinz - G. Kaymak - P. Ruggendorfer, ÖJh 68, 1999, Beibl. Jahresbericht 1998, $31 \mathrm{ff}$.

55 Die Untersuchung der Plinthe kann helfen, den bestechenden Ansatz, der von Fleischer a. O. $153 \mathrm{f}$. zur Zusammengehörigkeit von Statue und Sarkophag geäußert wurde, weiter zu präzisieren. Fleischer faßt beide Stücke als ikonographische Einheit auf und interpretiert sie gleichsam als dreidimensionale Umsetzung eines ostionischen Totenmahlreliefs. 


\section{ZuR ReKONSTRUKTION}

\subsection{Bisherige Rekonstruktionen und die sich daraus ergebenden Fragestellungen}

Seit der Rekonstruktion des Obergeschosses durch M. TheuER, welche ohne Zweifel stark von den Vorstellungen vom Maussolleion von Halikarnassos geprägt ist und eine weite Verbreitung gefunden hat, wurde aufgrund des angewachsenen Denkmälerbestandes und des in den letzten Jahren verstärkten Interesses an der frühhellenistischen Architektur die Frage nach der Wiedergewinnung der ursprünglichen Gestalt des Mausoleums wiederholt in das Zentrum bauhistorischer Diskussion gestellt ${ }^{56}$.

Theuers Variante des Mausoleums mit Pyramidendach, welche lange Zeit die Forschung dominierte, geht von einer typologischen Verbindlichkeit des Vorbildes aus und überrascht daher keineswegs (Abb. 7 ${ }^{57}$. Aufgrund fehlender architektonischer Evidenz für ein Pyramidendach hat er allerdings alternativ auch eine hypäthrale Lösung mit Innenhof entwickelt (Abb. 8) ${ }^{58}$. MartinI und Pinkwart sprachen sich zurecht dafür aus, das Monument von Belevi als eigenständiges Bauwerk zu betrachten und in dem Bau keine Kopie des Maussolleions von Halikarnassos zu sehen ${ }^{59}$. Der Alternativvorschlag THEUERS wurde jüngst von HoEPFNER aufgegriffen und überarbeitet (Abb. 9) ${ }^{60}$.

In der Rekonstruktion von HoEPFNER ist dem hypäthralen, rechteckigen Kernbau an der Außenseite der Nordwand eine zusätzliche Säulenstellung vorgeblendet, der die schon erwähnten Fragmente der Palmblattkapitelle und der Säulen mit geringerem Durchmesser sowie die Zwei-Fascien-Architrave mit den Inschriftenresten zugeordnet werden. Da die geringer dimensionierten Säulen auch eine geringere Höhe aufweisen, rekonstruiert er - um die durch die Peristasis vorgegebenen Höhe zu erreichen - an der Außenseite der Nordwand ein Bathron, auf dem diese zusätzliche Säulenstellung ruhte. In Analogie zum Pergamon-Altar ergänzt er sitzende Frauenstatuen in den Interkolumnien ${ }^{61}$.

Im Gegensatz zu Hoepfner wies Theuer die Zwei-Fascien-Architrave den Wandgliederungen im Inneren des Kernbaus zu. Diese Zuweisung blieb aber rein hypothetisch - so waren z. B. für MARTINI keine „konkreten Argumente für die Annahme der ungewöhnlichen, verkröpften, zweigeschossigen Säulengliederung im Inneren" ersichtlich ${ }^{62}$.

Auch Pinkwart geht von einer hypäthralen Anlage aus. Aufbauend auf dem Steinplan THeuERs entwickelte sie einen Altarhof mit teilweise offener Südseite und vorgelagerter doppelter bzw. einfacher Säulenstellung mit davorliegender Plattform ${ }^{63}$. Den Innenseiten des Altarhofes weist sie eine Fassadengestaltung mit verkröpftem Gebälk zu.

Die Frage nach Gliederung und architektonischer Ausformung sowohl der Innen- als auch der Außenseiten der Wände des Kernbaus im Obergeschoß, die eng verbunden ist mit der Frage nach seiner exakten Positionierung und Ausdehnung, stellt eines der Hauptprobleme unserer Arbeiten dar.

56 Praschniker - Theuer 69 ff.; Hoepfner 122.

57 Praschniker - Theuer 72f. Abb. 51. 52.

58 Praschniker - Theuer 57 Abb. 42a; so auch Pinkwart $764 \mathrm{ff}$.

59 Martini 73; Pinkwart 772.

${ }^{60}$ Hoepfner $111 \mathrm{ff}$.

${ }^{61}$ Hoepfner $116 \mathrm{ff} .120$.

62 Martini 73. Die Aufnahme der Elemente des Obergeschosses ist in Arbeit.

63 Pinkwart 767 ff. Abb. 1, 2. 
Die Höhe des Sockels setzt HoEPFNER tiefer an als THEUER ${ }^{64}$. Er begründet dies mit der Problematik der Entwässerung des hypäthralen Innenhofes und schlägt deswegen eine Peristasis vor, die tiefer als der Innenhof liegt ${ }^{65}$. Dadurch verändern sich die Proportionen des Monumentes beträchtlich.

Durch präzise Aufnahme aller technischen Details am Sockel selbst und an seinen dislozierten Architekturgliedern konnte die Grundlage für die Ermittlung der exakten Sockelhöhe und des Schichtaufbaues geschaffen werden, welche für alle weiteren Überlegungen hinsichtlich der Gesamtproportionen des Monumentes und aller damit in Zusammenhang stehenden Detailfragen die Ausgangsbasis darstellt.

Neben der Überdeckung des Obergeschosses stellen sich hier die Fragen bezüglich des Grades und der Art der Unfertigkeit, auch deshalb weil nicht auszuschließen ist, daß eine Entwurfsänderung während der Bauausführung anzunehmen ist. Auch die des öfteren diskutierte Annahme mehrerer Bauphasen $^{66}$ stellt ein noch zu lösendes Problem dar.

Die Untersuchung der verschiedenen Ausarbeitungsstadien der Architekturelemente und der Bauornamentik greift in die Beantwortung dieser Frage mit ein. Darüber hinaus sind Untersuchungen zur Metrologie des Baus, zu verschieden dimensionierten Interkolumnien, leicht variierenden Schichthöhen und daraus abzuleitenden perspektivischen Wirkungen noch ausständig. Erst diese Untersuchungen werden den Besonderheiten des Baus und seinen bautechnischen Details gerecht werden.

\subsection{Die vorbereitende Bauforschung}

Angesichts der Vielzahl der ungeklärten Fragen wurde in der Kampagne 1998 untersucht, inwieweit die bisherigen Dokumentationen und Aufnahmen eine Basis für die zukünftigen Forschungen bilden können.

Die Autopsie der Befunde in der direkten Gegenüberstellung mit dem bisherigen Planmaterial von Theuer (überarbeitet von SchotTenhaml und Fossel-Peschl) zeigte deutlich, daß diese Zeichnungen, die auch die Basis für HoEPFNERS Rekonstruktion bildeten, als Grundlage für theoretische Rekonstruktionen nicht ausreichen und in wesentlichen Punkten einer Ergänzung bzw. Neuaufnahme bedürfen ${ }^{67}$.

Sowohl im publizierten als auch im unpublizierten Planmaterial im Archiv des Österreichischen Archäologischen Institutes (ÖAI) in Wien ist v. a. die in situ befindliche Architektur an der Oberseite und an den Seitenflächen des Sockels nur sehr schematisch dokumentiert. Die Pläne reichen nicht einmal zur theoretischen Ermittlung der Höhe und des Schichtenaufbaues des Sockels aus.

${ }^{64}$ Hoepfner 115 Abb. 10, lt. Text um 1,95 m tiefer, lt. Rekonstruktionszeichnung um ca. $1,50 \mathrm{~m}$ tiefer.

65 Hoepfner 115. Die Ableitung des Regenwassers bedingt entweder einen Plattenboden des Innenhofes, der höher liegt als die Peristasis, oder einen Abfluß, der unter der Ringhalle durchführt. Da Hoepfner für einen solchen Abfluß im Felskern kein Hinweis erkennen konnte, wählt er die erste Variante.

66 Vgl. W. Alzinger in: Praschniker - Theuer $199 \mathrm{f}$.

67 Dies soll und darf die Leistungen der älteren Forschungen in keiner Weise schmälern. Hier wurden sowohl auf dem Bereich der Archäologie als auch der Bauforschung trotz arger finanzieller und zeitlicher Begrenzungen von allen Tätigen wertvolle Grundlagen erarbeitet. 


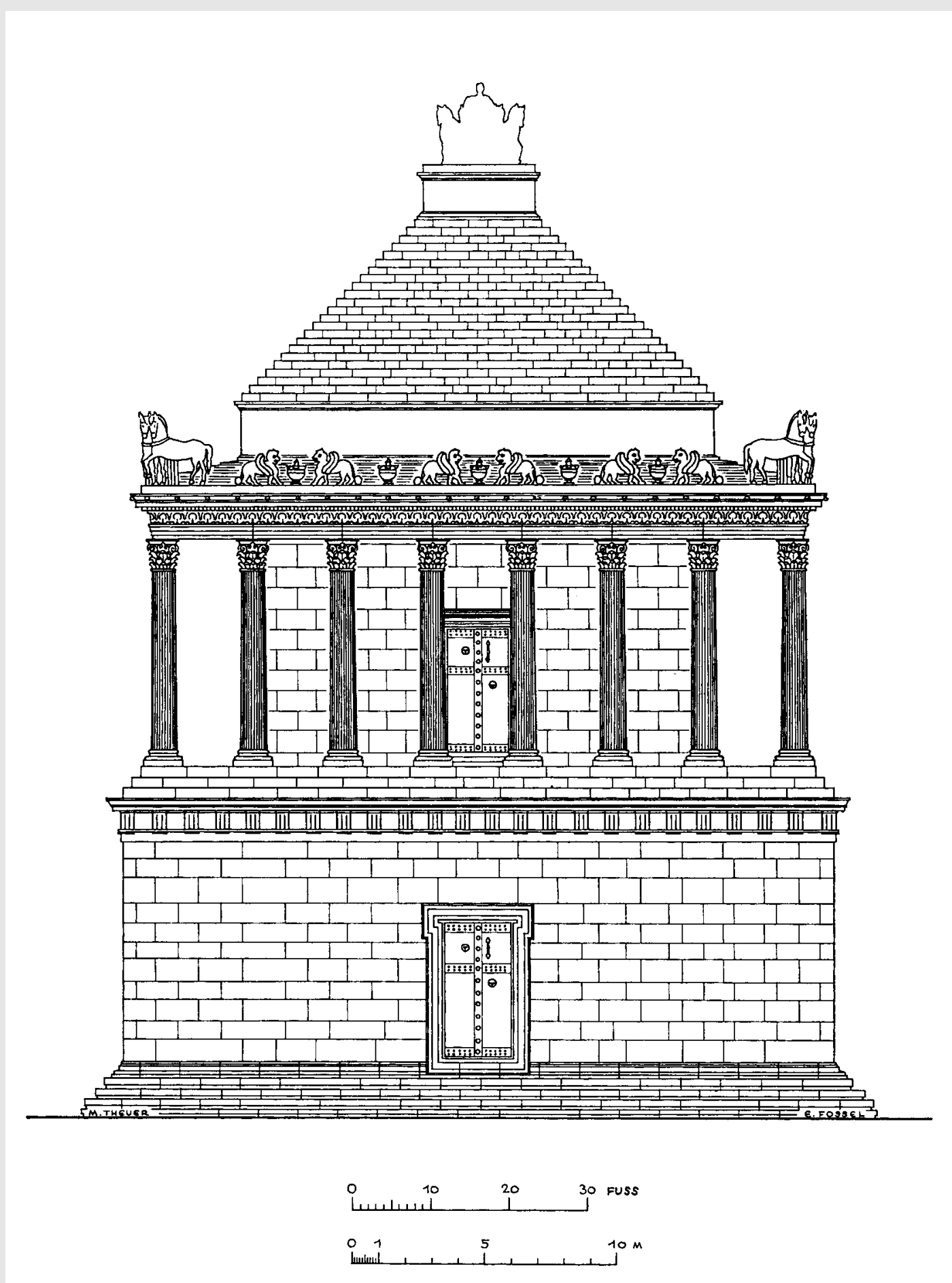

41. 77 E.FOSSEL

Abb. 7: Nordansicht in der Rekonstruktion nach Theuer 

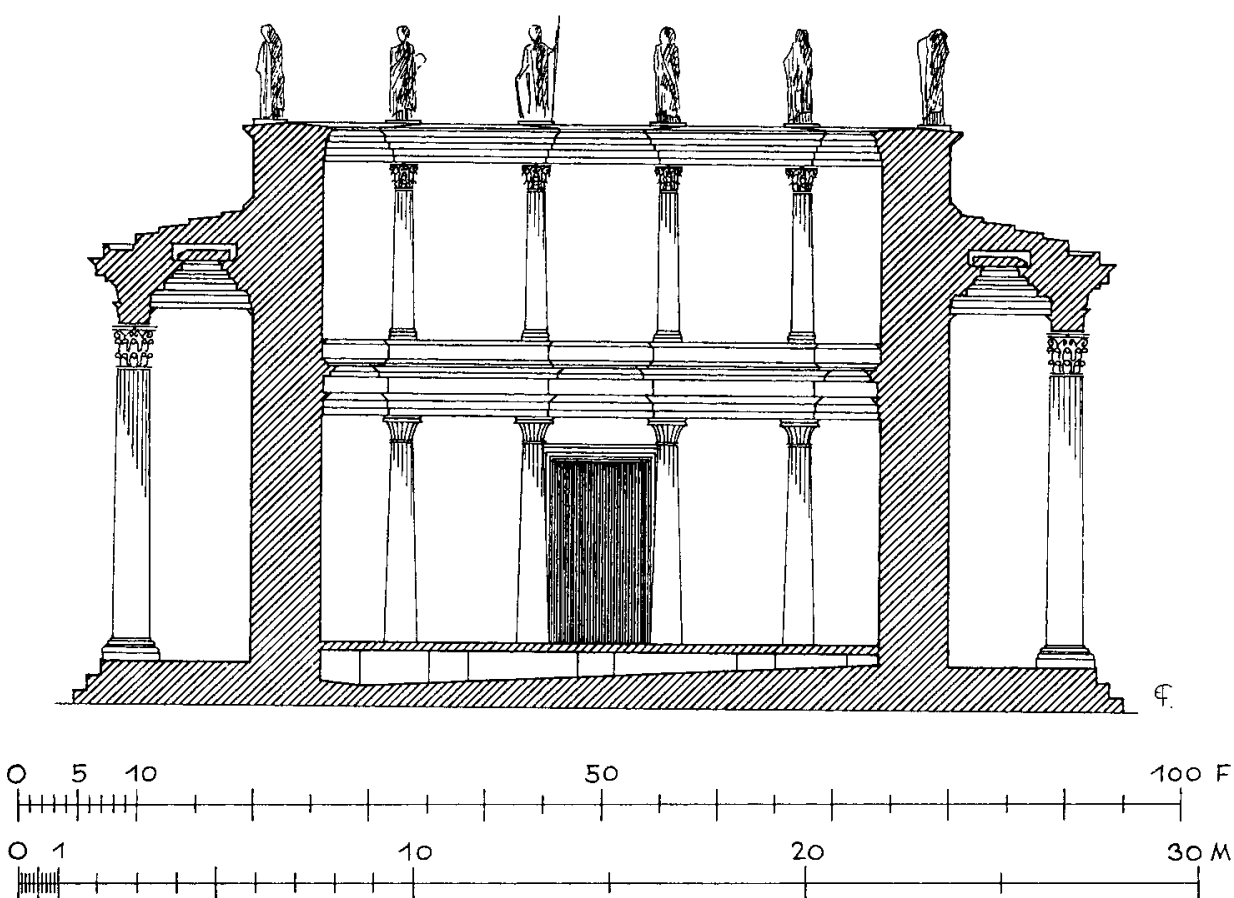

Abb. 8: West-Ost-Schnitt durch das Obergeschoß nach einem Rekonstruktionsversuch von Theuer
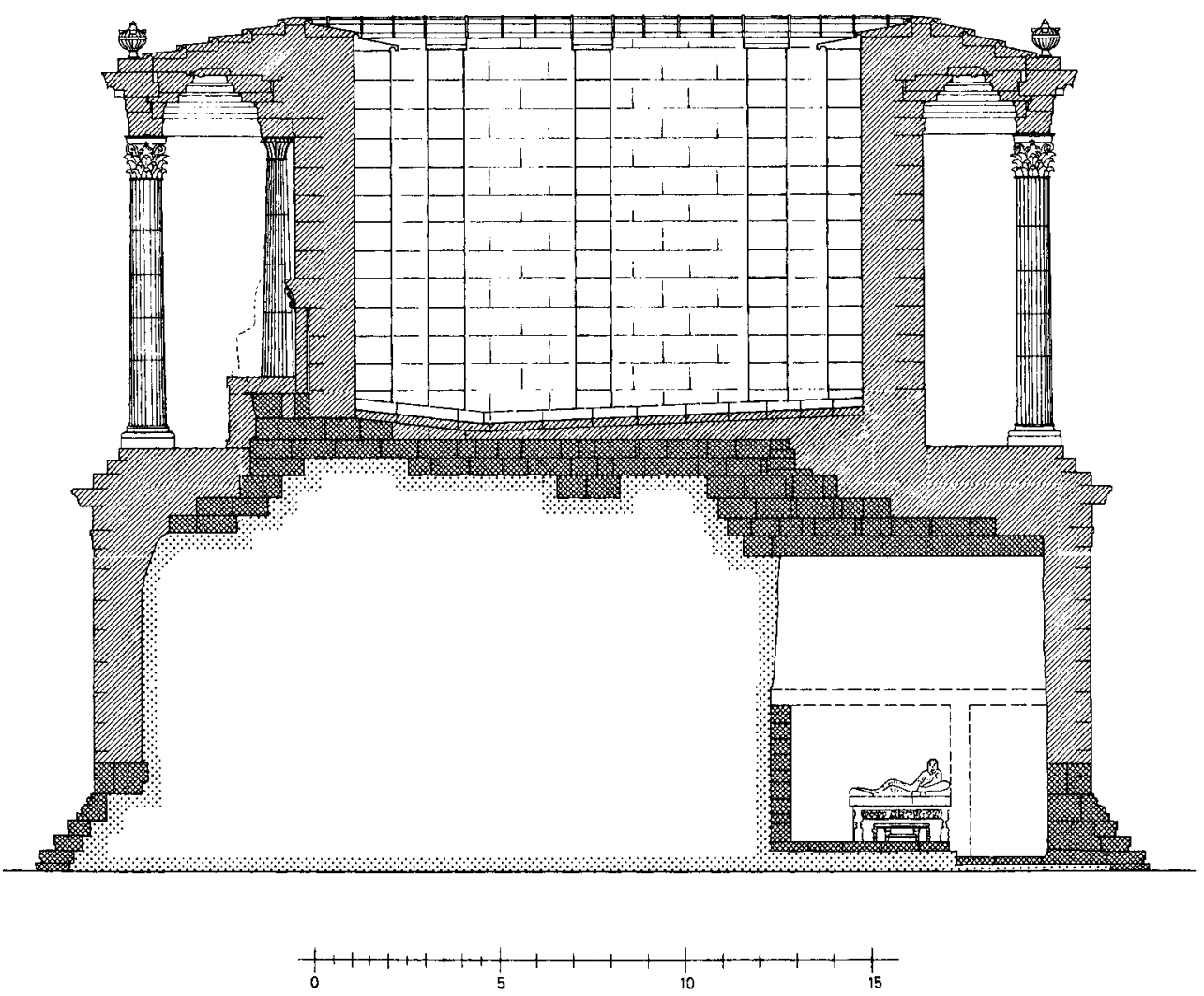

Abb. 9: Nord-Süd-Schnitt in der Rekonstruktion nach Hoepfner 
Grundsätzlich widmeten sich unsere Arbeiten der Dokumentation des in situ Bestandes, der Neuaufnahme des Sockels und einer exakten Bauaufnahme der dislozierten Bauglieder.

$\mathrm{Zu}$ diesem Zweck wurde mit der steingerechten, verformungsgetreuen Neuaufnahme des Sockels begonnen. Grundlage der Aufnahme bilden händisch erstellte Zeichnungen im Maßstab 1:10. Diese Zeichnungen wurden digitalisiert und mit CAD- und Bildbearbeitungsprogrammen weiterbearbeitet, mit ergänzenden Informationen ausgestattet und in ein CAD-gestütztes Dokumentationsmodell übernommen (Abb. 10). Aus dem zentralen Modell lassen sich Detailinformationen entnehmen, die zur Erstellung von Schichtenplänen und als Grundlage und Ausgangspunkt für die theoretische Rekonstruktion dienen.

Als Unterstützung für die händischen Aufnahmen wurden Teilbereiche der Nordfront photogrammetrisch aufgenommen. Die Aufnahmen werden händisch ergänzt und überarbeitet.

Die genaue Dokumentation des in situ-Bestandes setzte an der Oberseite des Sockels an. Im Zuge der Grundrißaufnahme der erhaltenen Oberfläche konnten im Südwesten drei Blöcke eindeutig als in situ befindlich erkannt und einer gemeinsamen Schicht im Unterbau des Bodenbelages zugeordnet werden $^{68}$.

Die Oberseiten dieser Blöcke sind zweifach geneigt. Einerseits weisen alle ein Hauptgefälle nach Norden auf, andererseits neigen sich die Flächen zusätzlich zu einer Ichse auf dem mittleren Block. Dieser Befund kann auch an den gegenüberliegenden Blöcken im Norden vor den Resten aufgehender Architektur des Kernbaus beobachtet werden ${ }^{69}$. Die Lage der Ichse wird hier durch eine fein ausgearbeitete Linie angedeutet, die den Verlauf des Anschlusses der Fußbodenplatten an das aufgehende Mauerwerk angibt. Die Lage der Blöcke im Südwesten ermöglicht zudem Rückschlüsse auf die Ausdehnung des Kernbaus und den ursprünglichen Verlauf der Gefälleebenen. Höhenlage und Ausmaß der Neigungen der nördlichen und südlichen Blöcke erfordern im mittleren Bereich des Kernbaus die entsprechenden Gegengefälle. Es ist also vermutlich mit zwei Abläufen im Westen zu rechnen. Die Klärung des Gefälleverlaufes trägt dazu bei, die Probleme der Entwässerung des Obergeschosses zu lösen.

In einem nächsten Schritt wurden die Mauerverläufe des Kernbaus untersucht.

Im Westen konnte an einem Pfeilerblock, der zu den Resten der aufgehenden Architektur des Obergeschosses zu zählen ist, eine bearbeitete gekrümmte Fläche festgestellt werden, die möglicherweise als Ansatz einer Halbsäule interpretiert werden darf. Zwei große Blöcke mit speziellem Zuschnitt konnten an der Ostseite als Teil des Unterbaus für zwei verlorene Wandpfeiler identifiziert werden. Die ungefähre Lage eines weiteren Wandpfeilers ergab sich im südlichen Teil der Westseite aufgrund grober Einarbeitungen im Felsen. Dadurch können nun präzisere Angaben zur Innengliederung des Obergeschosses gemacht werden.

Parallel dazu wurde mit der Aufnahme der technischen Anschlüsse bzw. der Abarbeitungen an den Seitenflächen des Felssockels begonnen, aus denen sich das System zur Verankerung der Verkleidungsquader und zur Aufnahme

${ }^{68}$ Einer der Blöcke ist nur geringfügig aus seiner originalen Lage verdreht.

69 Vgl. zur Struktur des Unterbaus des Fußbodens im Nordbereich die Angaben von Hoepfner 114. 


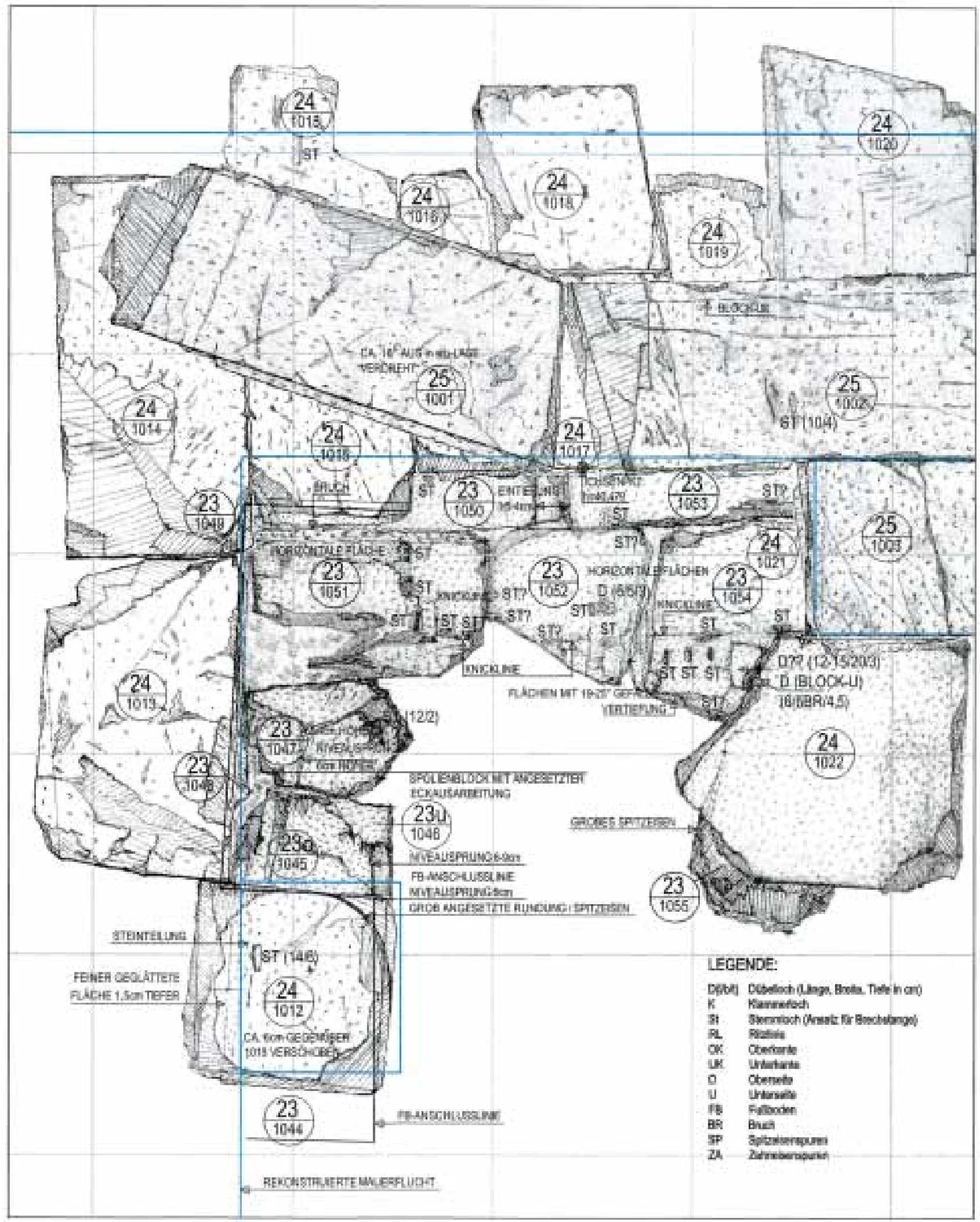

MAUSOLEUA WON BELEN AUSSCHNITT BESTANDSPLAN

Abb. 10: Ausschnitt aus dem Bestandsplan 2000 von NW-INNENECXE KEPQVANU der nordwestlichen Innenecke des Kernbaues

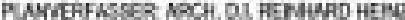

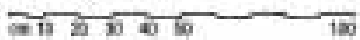




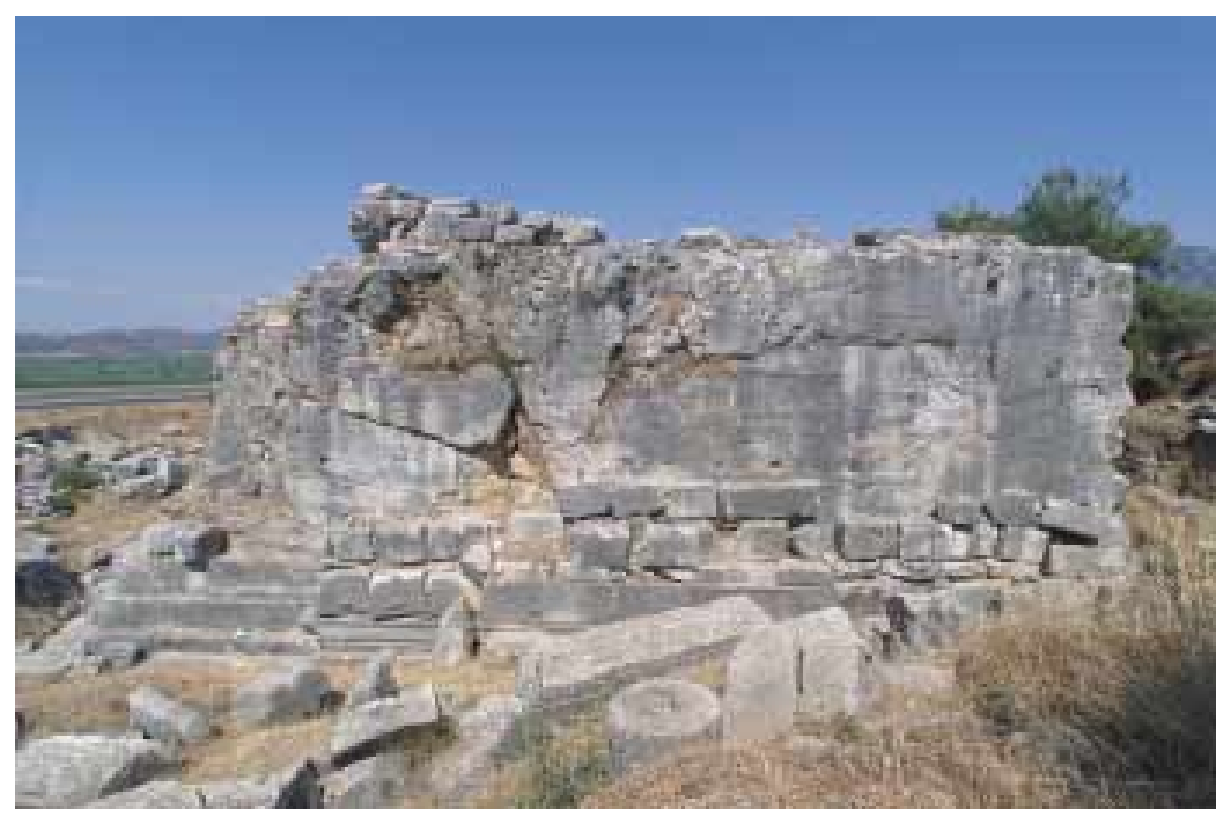

Abb. 11: Westansicht des Mausoleums

der Lagerfugen ablesen läßt (Abb. 11). Neben den Höhen der in situ verbliebenen Blöcke des Untergeschosses ergeben sich daraus Hinweise auf den Schichtaufbau ${ }^{70}$, die in einem Schichthöhenplan zusammengefaßt wurden. Dadurch ergibt sich ein relativ klares Bild einer variierenden Schichtabfolge von hohen Blöcken, zwischen denen jeweils niedrigere eingeschoben sind. Dazu kommt, daß diese Schichthöhen von unten nach oben kontinuierlich abnehmen. Bei den am Felskern gewonnenen Daten zur Höhe der Lagerfugen muß wegen der Entfernung zur Außenfront mit größeren Toleranzen gerechnet werden. Diese konnten jedoch durch die Vervollständigung und Ergänzung über die Maße der dislozierten Verkleidungsblöcke minimiert und ausgeglichen werden, so daß nun präzise Aussagen zur Schichtabfolge bis zum Obergeschoß und zur Höhe des Sockels möglich sind (Abb. 12) ${ }^{71}$ :

Die Sockelhöhe von der Unterkante der ersten Stufe des Untergeschosses bis zur Oberkante des Stylobates des Obergeschosses konnte so mit $10,70 \mathrm{~m}$ bestimmt werden $^{72}$. Sie läßt sich direkt an einer schmalen Erhöhung am abgearbeiteten Fels in der Nähe der Südwestecke ablesen. Diese Felsbank trägt eine Anathyrosis und ein rundes Dübelloch - Befunde, die sonst nicht im derzeitigen Bestand des Obergeschosses vorkommen und immer in Zusammenhang mit knapp an der ursprünglichen Oberfläche liegender exakter Ausarbeitung stehen (Abb. 11). Die Sockelhöhe ist geringer als jene in den Rekonstruktionen von Theuer und von HoEPFNER ${ }^{73}$. Als Ergebnis lassen sich 19

70 Vgl. Praschniker - Theuer 13.

71 Vgl. Praschniker - Theuer 15. Theuer vermutet, daß die Schichtabfolge an den vier Seiten nicht gleich war, da die Klammerlöcher im Fels in unterschiedlicher Höhe angebracht sind. Die unterschiedliche Höhe erklärt sich daraus, daß die Klammern einmal an der Oberseite einer schmalen Schicht, dann wieder an der Oberseite der darunterliegenden hohen Schicht angebracht wurden. Den Wechsel der Schichthöhen von hoch auf niedrig negiert Theuer.

72 Vgl. ÖJh 70, 2001, Beibl. Jahresbericht 2000, 263 f.

73 Praschniker - Theuer 69f. Abb. 50: UK 1. Stufe bis OK Stylobat = 12,793 m, lt. Hoepfner Abb. 10 ca. 11,20 m. 


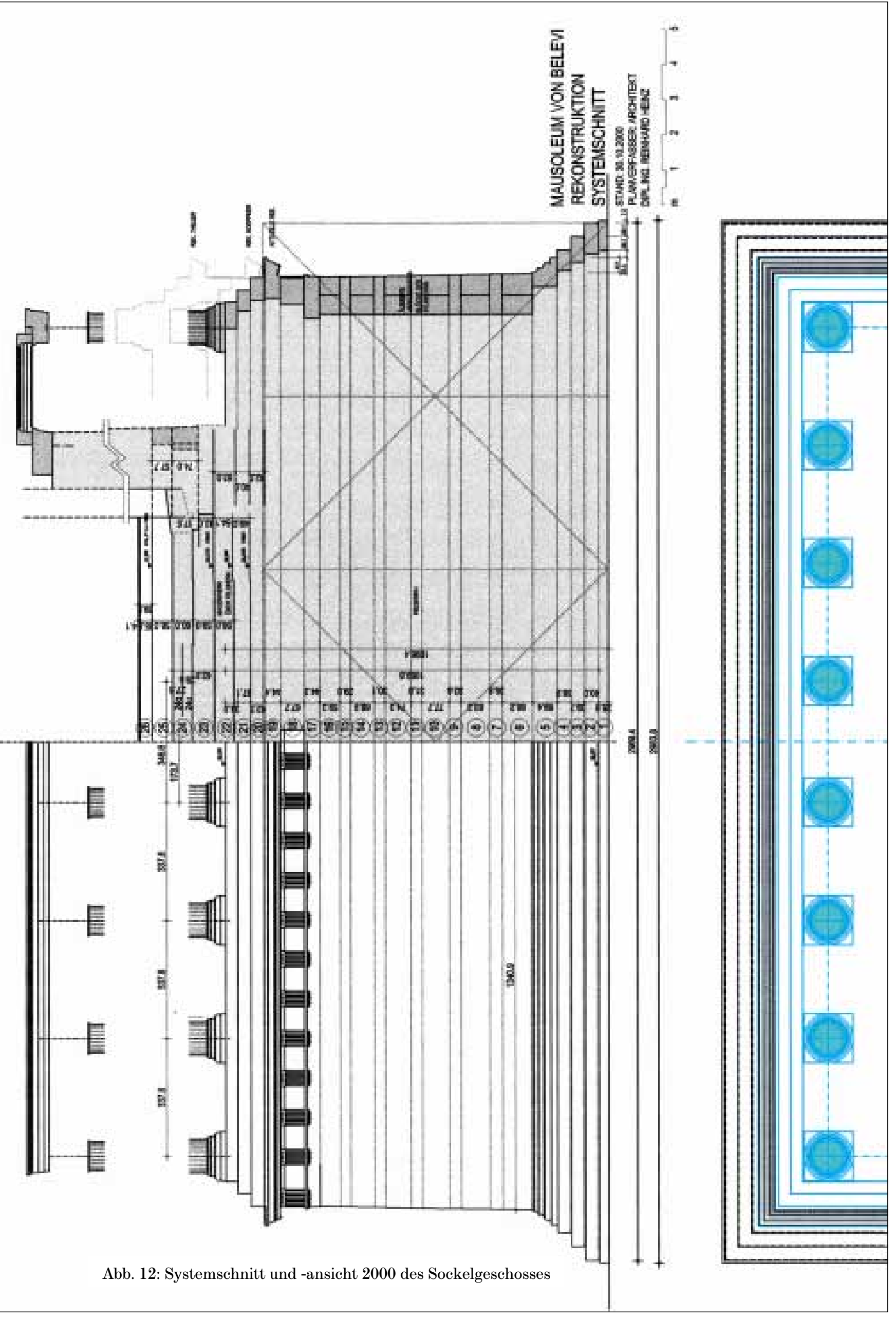


Blockschichten bis zum dorischen Gesims des Sockels ermitteln. Der oberste in situ befindliche Block des aufgehenden Mauerwerks der Kernbaunordwand ist der Schicht 26 zuzuordnen.

Bei der Untersuchung des Sockels konnte beobachtet werden, daß der Zwischenraum zwischen den Verkleidungsblöcken und dem Felsen mit Bruchsteinen und Kalkmörtel hinterfüllt ist. Da dieser Befund an allen Seiten sowohl an den Wänden des Felssockels als auch im Bereich des Stufenunterbaus, am darüberliegenden Sockelprofil und der ersten in situ verbliebenen Schar Verkleidungsblöcke angetroffen wurde, kann mit Sicherheit gesagt werden, daß es sich nicht um eine römische Reparatur oder um eine sekundäre Baumaßnahme handelt, sondern hier eine Technik nachgewiesen ist, die bei der Errichtung des Baus konsequent zum Einsatz kam (Abb. 13) ${ }^{74}$. Die Untersuchung sämtlicher an diesem Monument vorkommender Mörtelarten stellt somit ein weiteres Desiderat für die zukünftigen Forschungen dar.

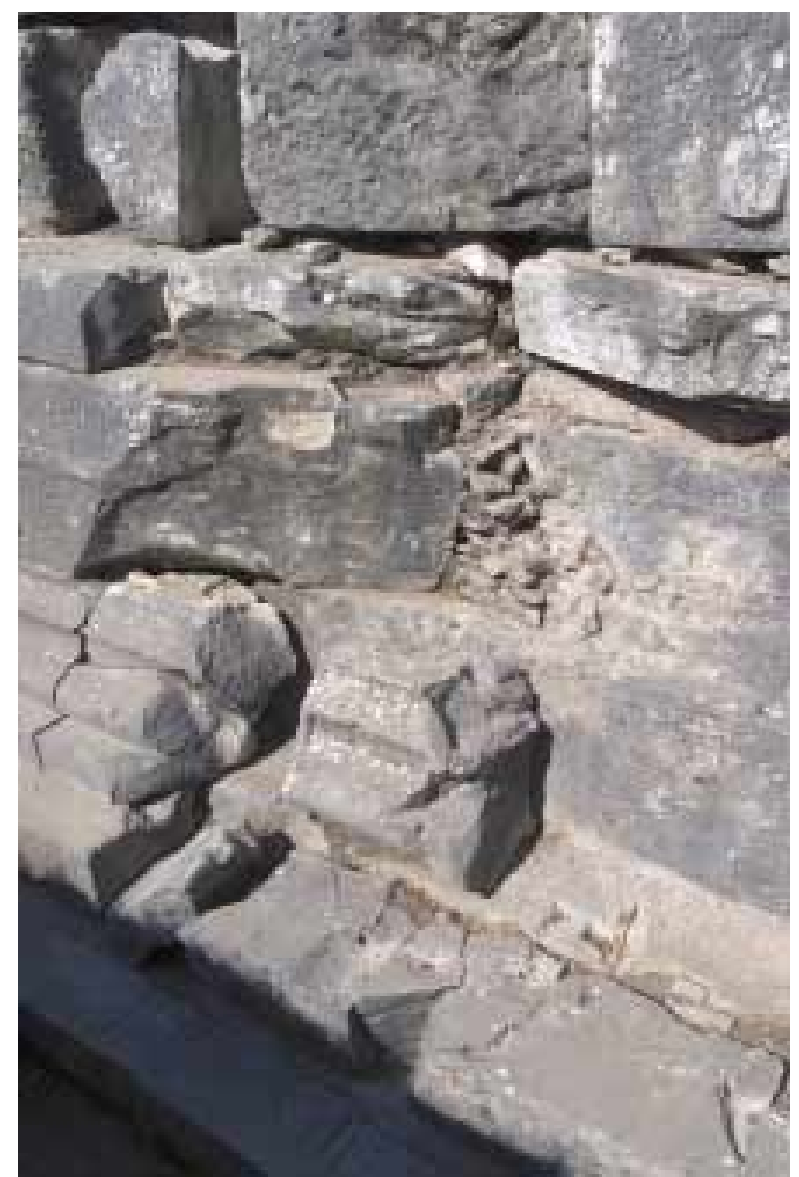

Abb. 13: Mörtelpackung hinter einem der untersten Wandverkleidungsblöcke an der Westfront.

${ }^{74}$ Auf der östlichen Terrasse wurde in der SO 1 neben dem nördlichen Kanal gleichfalls ein schmales Mörtelband angetroffen, das mit feinem Steinsplitt durchsetzt ist und in seiner Zusammensetzung große Ähnlichkeit mit dem am Mausoleum verwendeten Mörtel besitzt. Dieser Befund kann dahingehend interpretiert werden, daß man den bei den Terrassierungsarbeiten anfallenden Steinsplitt gesammelt, diesen gleich an Ort und Stelle mit Mörtel vermischt und dann umgehend am Mausoleum verbaut hat. 
Die Kassettenreliefs im Museum von Izmir wurden einer Autopsie unterzogen und dabei die Position und Zuordnung jeder Platte unter Zuhilfenahme der Fundortangaben aus den Originalgrabungstagebüchern und besonders anhand der an den Blöcken erhaltenen technischen Informationen überprüft. An der Zuweisung der Platten und Plattenfragmente an die jeweiligen Seiten darf zum derzeitigen Forschungsstand zur Ikonographie festgehalten werden. Unklarheiten bestehen jedoch bei der Positionierung der Platten innerhalb der einzelnen Seiten und vor allem an den Eckbereichen $^{75}$.

Aufgrund dieser neuen Untersuchungen wird sich die architekturhistorische Einordnung des Mausoleums in neuem Licht darstellen lassen und die modifizierte Rekonstruktion wird neue metrologische Überlegungen ermöglichen, die diesem Monument eine einmalige Position zwischen Spätklassik und Hellenismus zuweisen.

\section{Datierung}

Zur Datierung des Mausoleums wurden bislang vorrangig die wenigen, während der Grabungen gefunden Keramikfragmente und die Bauornamentik herangezogen.

Das Keramikmaterial ist insgesamt spärlich und setzt sich aus zwei Fundkomplexen zusammen: Der erste konnte in der Grabkammer außerhalb des Sarkophages geborgen werden und ist in den Zeitraum vom Ende des 1. vor- bis zum Ende des 1. nachchristlichen Jahrhunderts zu datieren ${ }^{76}$.

Der zweite - „ein Nest aus Scherben“ - wurde nahe dem Fußboden an der Westseite des Baus innerhalb einer Anhäufung von Marmorsplittern ergraben. Diese Fragmente datieren mehrheitlich in den Zeitraum zwischen ca. 350 und 300 v. Chr. ${ }^{77}$ Nur zwei Fragmente, ein Amphorenfuß und das Schulterfragment eines Kantharos, stammen aus der Zeit ungefähr um 280 v. Chr., wobei das Kantharosfragment in der Fundbeschreibung von PraschNiker nicht erwähnt wird und seine Zugehörigkeit zum Keramikmaterial des Mausoleums daher mit Vorbehalt betrachtet werden muß ${ }^{78}$.

Das „Nest aus Scherben“ umfaßt ausschließlich Gebrauchskeramik und wird zur Zeit als Material interpretiert, das von Bauhandwerkern benutzt wurde. Dafür könnte auch die Fundlage und der Fundort der Fragmente auBerhalb der Grabkammer vor dem Stufenunterbau an der Westseite des Mausoleums sprechen ${ }^{79}$.

75 Zur Zeit werden die Eckplatten der Nord- und der Südseite zugeordnet. Diese würden demnach über jeweils 7, die West- und die Ostseite jeweils über 5 Kassettenreliefs verfügen, vgl. Praschniker - Theuer $73 \mathrm{ff}$. Zur Problematik der Platte an der Südwestecke kann nach Praschniker die Möglichkeit einer falschen Versetzung weiter eingeengt werden.

${ }^{76}$ Vgl. V. Mitsopoulos in: Praschniker - Theuer $161 \mathrm{ff}$.

77 Praschniker - Theuer 106. $161 \mathrm{ff}$.

78 Mitsopoulos a. O. $161 \mathrm{ff}$. Vgl. zur Keramikdatierung die skeptische Haltung von Pinkwart 770. Vgl. die mit Zurückhaltung zu wertende Datierung, die Bauornamentik und Keramik zu korrelieren versucht, von Rumscheid 76: „Dieses Ergebnis paßt zur Datierung der jüngsten Scherben um 280 v. Chr. ..."

79 Mitsopoulos a. O. 166. Rumscheid 76. Die Keramik wird im Rahmen dieses Projektes von H. Liko gemeinsam mit den Neufunden einer Neubewertung unterzogen. 
In der Bauornamentik sind im allgemeinen große Ähnlichkeiten zu Bauten feststellbar, die in ihrer zeitlichen Stellung fest ins 4. Jh. v. Chr datiert sind, wie z. B. zum Andron B und dem Zeus Tempel sowie den Südpropyläen in Labraunda, besonders aber zum Athena-Tempel von Priene ${ }^{80}$.

Nach RumscheID sprechen „,ie Detailformen der lesbischen Kymata und verschiedene Parallelen am Arsinoeion für eine Datierung erst ins 3. Jh. etwa in die Zeit zwischen 290 und $270 v$. Chr. "81 Auffallend sind bei den Kymatienformen auch die Bezüge zum „Heroon“ auf der Anhöhe 84 in Demetrias, das wohl gleichfalls an den Beginn des 3. Jhs. v. Chr. zu datieren ist ${ }^{82}$.

Der figürliche Schmuck am Bau wird dem Forschungsstand entsprechend allgemein ins 3. Jh. v. Chr. datiert, wobei aber im Detail von einer genaueren Einordnung Abstand genommen wird ${ }^{83}$. Die gelagerte Deckelfigur des Klinensarkophages gilt in vorrömischer Zeit als singulär. Aus der von Fleischer unternommenen Überlegung - Sarkophag und Dienerfigur ergeben gemeinsam die freiplastische Parallele zu ostionischen Totenmahlreliefs leiten wir eine chronologische Überlegung ab, die in das frühe 3. Jh. v. Chr. weist. Der Diener ist orientalisch gekleidet, der Grabherr ist offenbar in einem hellenistischen Herrscherornat dargestellt. Weitere Begleitfiguren fehlen. Auf einem heute verschollenen Elfenbeinrelief aus Demetrias mit der Darstellung eines Totenmahls sind die Figuren ebenfalls in persischer Tracht gekleidet. Es wird in hellenistische Zeit datiert ${ }^{84}$.

\section{Die Frage Nach dem GrabherRn}

Die Datierung der Bauornamentik und der Keramik legt nahe, an eine Persönlichkeit aus der Zeit des ausgehenden 4. Jhs. v. Chr. bzw. des frühen 3. Jhs. v. Chr. zu denken.

Zur Identifizierung des Grabherrn wurden bislang in Ermangelung eindeutiger Information vom Bau selbst ausschließlich historische Indizien herangezogen, wobei die Tatsache, daß das Monument offensichtlich nicht vollendet wurde, ein zentrales Argument darstellt.

Diskutiert wurden neben Lysimachos und Antiochos II. Theos auch der General Mentor von Rhodos, der 336 v. Chr. starb, und sein kurz nach ihm verstorbener Bruder Memnon ${ }^{85}$.

80 Zuletzt Rumscheid 70 ff. Vgl. die Kritik von Pinkwart 765 an den von W. Alzinger in, Praschniker - Theuer $177 \mathrm{ff}$. zusammengestellten Vergleichsmonumenten aus dem 4. - 2. Jh. v. Chr. Kritisch dazu auch Martini 74: „Datierung und Zuweisung an einen Bauherrn bzw. Grabinhaber stehen seit jeher in so enger Wechselbeziehung, daß nach Praschniker häufig den historischen Erwägungen der datierende Vorrang gegeben wurde. Das äußert sich z. B. auch in der stilistischen Beurteilung der Bauornamentik durch Alzinger (17\%ff.), der zwar mit Praschniker den spätklassischen Einflu $\beta$ sieht, aber doch eine - freilich nicht meßbare - größere Nähe zu hellenistischen Formen annimmt. Der Frage nach den Gründen für die offensichtliche starke Bindung an den spätklassischen Formenschatz wird nicht nachgegangen."

81 Rumscheid 76. Zum Vergleich mit dem Ptolemaion von Limyra s. J. Borchhardt G. Stanzl in: Götter, Heroen, Herrscher in Lykien. Ausstellungskatalog Schallaburg (1990) 80; J. Borchhardt, Die Steine von Zêmuri (1993) $79 \mathrm{f}$.

82 P. Marzolff in: H. Hauptmann (Hrsg.), Demetrias V (1987) $26 \mathrm{ff}$.

83 Vgl. Martini 74.

${ }^{84}$ H. Kyrieleis, Throne und Klinen. Studien zur Formengeschichte altorientalischer und griechischer Sitz- und Liegemöbel vorhellenistischer Zeit, 24. Ergh. JdI 1969, 149 Taf. 18, 3.

85 R. Fleischer und W. Alzinger in: Praschniker - Theuer $156 \mathrm{ff} .193$. 
In der neueren Forschung wurde verstärkt Lysimachos der Vorzug gegeben, der Alt-Ephesos verlegt und unter dem Namen seiner Frau und späteren ptolemäischen Königin Arsinoe II. als Arsinoeia neu gegründet hat ${ }^{86}$. Die Schwierigkeiten bei der Gleichsetzung des Mausoleums von Belevi mit dem Grabmal des Lysimachos sind evident ${ }^{87}$ : Lysimachos, der 281 v. Chr. in der Schlacht von Kouropedion gefallen war, wurde nach Aussage der philologischen Quellen in Lysimacheia auf der thrakischen Chersones bestattet ${ }^{88}$.

Nach der Theorie der Erstbearbeiter wären die Arbeiten am Mausoleum nach dem Tod des Lysimachos vorerst eingestellt worden. In einer weiteren Bauphase und nach einer Änderung des ursprünglichen Entwurfs wären sie aber nach Tod des Antiochos II. Theos in Ephesos im Jahre 246 v. Chr. fortgesetzt worden, da keine Möglichkeit bestand, den Leichnam des seleukidischen Königs nach Antiochia zu überführen. Das Mausoleum hätte nun als Grabmal des Antiochos II. Theos gedient ${ }^{89}$. HoEPFNER hingegen lehnt diese Theorie der zwei Bauphasen $a^{90}$. Ein Weiterbau des Mausoleum für Antiochos II. Theos wäre „undenkbar, da die Ephesier nach seinem Tode (Tod des Lysimachos, Anm. der Verf.) die Erinnerung an den ungeliebten König auslöschen wollten" $"$.

Die Frage nach dem Grabherrn ist in der Sepulkralarchitektur von zentraler Bedeutung. Er und seine Intentionen der Selbstdarstellung geben über sein Ableben hinaus dem Grabbau Anlaß und Bedeutung. Deshalb ist auch die Frage nach dem Grabherrn von Belevi von entscheidender historischer Wichtigkeit. Durch eine methodisch klare Abwägung der archäologischen, bauhistorischen und kunsthistorischen Argumentationen versucht dieses Projekt, eine neue Antwort zu finden.

86 Für Lysimachos haben sich Hoepfner 123 und (mit Einschränkungen) Rumscheid 76 ausgesprochen. Zur Diskussion über Mentor und Memnon von Rhodos s. W. Alzinger in: Praschniker - Theuer $190 \mathrm{ff}$.

87 Ausführlich Alzinger a. O. 191.

88 Paus. 1, 10, 5; App. Syr. 64. Lysimacheia wurde - möglicherweise konzipiert als neue Hauptstadt - durch Synoikismos von Lysimachos im Jahre 309/08 v. Chr. gegründet, vgl. W. Leschhorn, Gründer der Stadt, Palingenesia 20 (1984) 257 (mit umfangreicher Lit.). Nach seinem Tod wurde sein Leichnam von seinen Gefolgsleuten dorthin verbracht und in einem Grabmal, das Lysimacheion genannt wurde, beigesetzt, vgl. Paus. 1, 10, 5; Leschhorn a. O. 257 Anm. 4.

89 Dem Ansatz folgten auch R. Fleischer und W. Alzinger in: Praschniker - Theuer 159. $199 \mathrm{f}$.

90 Hoepfner 122. So auch schon Pinkwart 767: „,... die für die zwei Bauphasen vorgebrachten Argumente (die das Regenwasser ableitende Pflasterung des Kernbaus, nicht genormte und nachlässig ausgeführte Dachziegel, fehlende Deckziegel und die Problematik von Sarkophagdeckel und -kasten, Anm. der Verf.) sind nicht überzeugend". Zurückhaltender hingegen Martini a. O. 74 : „Inwieweit es aber vielleicht doch zwei Bauphasen gegeben hat, bleibt unklar. ... Da allein die Unfertigkeit der Greifen und auch des einen korinthischen Kapitells noch keine 2. Bauphase begründet, bleibt nur für eine sekundäre Nutzung der Grabkammer der zweifellos nachträglich angefertigte, grobe Sarkophagdeckel; da es schwerfällt, im Porträt des auf dem Deckel Gelagerten hellenistische Züge zu entdecken (vgl. Praschniker 117), ist eine noch spätere Entstehung nicht ganz auszuschließen."

${ }^{91}$ Hoepfner 123. Hingegen hat R. Fleischer, Studien zur seleukidischen Kunst I. Herrscherbildnisse (1991) 25 weitere Überlegungen zu einer seleukidischen Phase am Mausoleum für den zweiten Band seiner Studien in Aussicht gestellt. 


\section{Abbildungsnachweis}

Abb. 1 Photo ÖAW, Photonr. B01/9397, Photographin A. Sulzgruber Abb. 2 Photo ÖAW, Photonr. B01/FN 2,4a, Photographin A. Sulzgruber Abb. 3 Photo ÖAW, Photonr. B01/FN 2,17a, Photographin A. Sulzgruber Abb. 4 Photo ÖAW, Photonr. B01/2114, Photographin A. Sulzgruber Abb. 5 aus FiE VI, Praschniker - Theuer, Abb. 119

Abb. 6 aus FiE VI, Praschniker - Theuer, Abb. 83

Abb. 7 aus FiE VI, Praschniker - Theuer, Abb. 51

Abb. 8 aus FiE VI, Praschniker - Theuer, Abb. 42a

Abb. 9 aus AA 1993, Hoepfner, Abb. 10

Abb. 10 ÖAW, Ausschnitt Bestandsplan 2000, Planverfasser R. Heinz

Abb. 11 Photo ÖAW, Photonr. B01/9334, Photographin A. Sulzgruber

Abb. 12 ÖAW, Systemschnitt 2000, Planverfasser R. Heinz

Abb. 13 Photo ÖAW, Photonr. B01/9232, Photographin A. Sulzgruber 